\title{
Chain dynamics in polymer melts at flat surfaces
}

Article

Accepted Version

Kirk, J. and IIg, P. (2017) Chain dynamics in polymer melts at flat surfaces. Macromolecules, 50 (9). pp. 3703-3718. ISSN 0024-9297 doi: https://doi.org/10.1021/acs.macromol.6b01943 Available at https://centaur.reading.ac.uk/70383/

It is advisable to refer to the publisher's version if you intend to cite from the work. See Guidance on citing.

To link to this article DOI: http://dx.doi.org/10.1021/acs.macromol.6b01943

Publisher: American Chemical Society

All outputs in CentAUR are protected by Intellectual Property Rights law, including copyright law. Copyright and IPR is retained by the creators or other copyright holders. Terms and conditions for use of this material are defined in the End User Agreement.

\section{www.reading.ac.uk/centaur}

\section{CentAUR}

Central Archive at the University of Reading

Reading's research outputs online 


\title{
Chain dynamics in polymer melts at flat surfaces
}

\author{
Jack Kirk and Patrick llg* \\ School of Mathematical, Physical and Computational Sciences, University of Reading, \\ Reading, RG6 6AX, UK \\ E-mail: p.ilg@reading.ac.uk
}

\begin{abstract}
We investigate, by extensive molecular dynamics simulations as well as a simplified single-chain model, the influence of steric hindrance on the dynamic properties of nonentangled chains in polymer melt due to confining surfaces. We extend the Rouse model to also include wall effects, using an additional potential that results from the assumption that chain conformations have reflected random-walk statistics, as first advocated by Silberberg. Results for end-to-end vector and Rouse mode correlation functions of chains end-tethered to the surface compare well with those obtained from molecular dynamics simulations of a multi-chain system using the Kremer-Grest beadspring model (KG MD). Even though the additional single-chain potential is parameterfree, we show that the accuracy of the model for surface chains is comparable to that of the Rouse model for bulk chains. An analytic dumbbell model accurately describes the longest Rouse mode correlation function of surface-tethered 'mushroom' chains immersed in a polymer melt at low grafting density. In addition, we find that a perfectly smooth surface enhances the influence of hydrodynamic visco-elastic coupling on the centre of mass motion near the surface.
\end{abstract}

\footnotetext{
*To whom correspondence should be addressed
} 


\section{Introduction}

Current interest in polymer dynamics at surfaces can be separated into two general system categories. In the first category, the size of the region in which chains are influenced by the surface is comparable to a bulk size. Examples of such systems are polymer melts or solutions in thin films or narrow pores. ${ }^{1}$ Also, systems with filler particles much larger than the size of polymer coils, and sufficiently high filler concentration, belong to this category. ${ }^{2,3}$ The global rheological properties will be strongly influenced by the chain behaviour near to the filler surfaces. Strong confinement occurs when there is no bulk region and any given chain may interact with more than one surface simultaneously, leading to different chain statics and dynamics compared to weak confinement, which is the focus of the current work, where chains only interact with a single surface and at least the static behaviour of chains in the surface vicinity coincides with an asymptotically wide channel.

In the second category, the only chain bounding surface lies at the system extremities, and the boundary layer is small compared to the bulk dimension. Then surface chain behaviour is relegated to a dynamic boundary condition which can be important for shear flows. ${ }^{4,5}$

For high molecular weight melts or dense solutions, a likely surface effect will be a change in the behaviour of entanglements. At the time of writing, investigations into the dynamics of entangled polymers in interfacial regions have concentrated on using equilibrium molecular dynamics simulations ${ }^{6-8}$ to find an 'entanglement molecular weight' parameter as a function of position relative to the surface, which may be inserted into a tube or slip-link model. The-

ories for entangled polymers are typically built upon the Rouse model, ${ }^{9}$ which in addition to low molecular weight systems, describes the dynamics of long entangled polymers on short time-scales before they are aware of the topological constraints formed from neighbouring chains. It is therefore to some degree fundamentally important to investigate how the nonentangled dynamics change near to surfaces, in order to identify the corresponding changes in entangled dynamics and the crossover between the two regimes.

In addition to entanglements, many recent molecular dynamics studies of surface chain dy- 
namics have been motivated by experimental observations that the glass transition temperature can change near to a surface. ${ }^{10-15}$ Both atomistically detailed ${ }^{10,11,13}$ and coarsegrained $^{14,15}$ simulation models have been used. Methods of investigation include probing changes in Rouse mode correlation functions or monomer layer occupation survival functions, ${ }^{13,14}$ and observing rates of diffusion parallel to and normal to the surface. ${ }^{14,15}$ A quite comprehensive review of older work was made by Mischler et al. ${ }^{12}$

In atomistic models, microscopic details not present in the coarse-grained descriptions, such as torsional potentials, may influence the particular wall interaction. However, even coarsegrained models, such as the Kremer-Grest Molecular Dynamics (KG MD) ${ }^{16}$ polymer melt model that we investigate in this work, may conserve important dynamic mechanisms occurring over the length scale of a single coarse-grained bond, whose strength depends on the particular choice of surface interaction and the parameters used in the interaction potentials. One such example which could be important at temperatures approaching the glass transition is 'layer-exchange dynamics', ${ }^{13}$ occurring between the identifiably ordered monomer layers induced by smooth surfaces. ${ }^{17}$ A recurrent issue which we aim to settle is the distinction between such microscopic surface effects, which depend strongly on chemical detail, and larger scale dynamics which are universal for all flexible polymers. Chain relaxation at long time scales is entropic in origin and depends more generally on how global chain orientation statistics differ near to a surface due to steric hindrance.

It has already been identified that the conformations of chains near a flat confining surface in a wide channel using the KG MD model are reasonably well described using reflected random walk statistics. ${ }^{18}$ In this work we construct a model for the Brownian dynamics of reflected random walks which reduces to the Rouse model in the bulk. Within this model we explore the dynamics of chains that are either end-tethered at the surface, or non-tethered but in the proximity of it, and compare the results with those of the KG MD model. We also consider how the inaccuracies of the Rouse model crossover to the modified-Rouse model. In recent years it has become more clear for exactly which observables and to what 
extent the Rouse model gives accurate predictions for general polymer melt systems. ${ }^{19-23}$ A joint experimental and simulation study of a $\mathrm{C}_{100} \mathrm{H}_{202}$ melt revealed deviations from the Rouse prediction for the dynamic structure factor in the high momentum transfer end of the considered spectrum, ${ }^{22}$ corresponding to the first three Rouse modes. In the simulation portion of the same study, correlation functions of even the longest Rouse modes displayed stretched exponentials, instead of the single exponential modes predicted by Rouse. Despite such discrepancies, the Rouse model can reasonably describe the bulk linear stress relaxation function of a wide variety of non-entangled polymer melts when the effects of glassy modes are taken account of. ${ }^{19}$

The Rouse model assumes the validity of Flory's hypothesis, ${ }^{24}$ which states that in the melt chain orientations satisfy random-walk statistics. Wittmer et al ${ }^{25,26}$ have described theoretically how the correlations of bond vectors within melt chains decay more slowly along the chain contour than predicted by Edwards. ${ }^{27}$ Resultantly, even in bulk there are nonnegligible deviations from Gaussian statistics up to length scales which may extend into the entanglement regime, as is the case for the KG MD model. There have been attempts to understand the relative success of the Rouse model despite this observation. ${ }^{28}$ In turn, the accuracy of other key Rouse assumptions have been considered: screening of hydrodynamic interactions, ${ }^{29,30}$ and the 'phantom-chain' picture in which topological interactions between neighbouring chains are neglected. ${ }^{31,32}$ Under strong confinement, approaching the two-dimensional and one-dimensional limit in polymer films ${ }^{33,34}$ and nano-tubes ${ }^{35}$ respectively, the Strasbourg group and co-workers have demonstrated much stronger deviations from ideality. These papers have shown that for ultra thin films of width comparable to a chain 'blob' length, there is a logarithmic correction to the chain dimension. Correspondingly it may be conjectured that a similar correction may occur in a wide channel for chains whose centre of mass falls within a single blob length from the surface. In this work we find that short flexible chains near to the surface are only weakly swollen in the surface parallel direction, and that this effect also has a contribution from a weak nematic interaction which 
occurs even for the 'fully-flexible' KG MD model. Although the ratio of confined radius of gyration to bulk radius of gyration is an increasing function of chain length, ${ }^{34}$ in weak confinement the distance from the surface to the centre of mass of surface chains scales with the bulk radius of gyration; ${ }^{18}$ therefore in wide channels corrections to ideality near the surface may be of even less importance for longer chains.

Comparison of the Rouse model with a multi-chain 'soft MD' model, which allows bond crossings and therefore explicitly satisfies the phantom-chain picture of the Rouse model, has been shown to fail to match the Rouse prediction for mid-bead mean square displacement. ${ }^{36}$ In the same work, the more realistic KG MD model, which does not allow bond crossings but has very similar conformation statistics to soft MD, showed improved agreement. This suggested that topological interactions play a dynamical role even before the onset of the entanglement regime, which for certain observables may even improve agreement with the Rouse model. In section 4 we compare results for mid-monomer and centre of mass mean square displacement near the surface using the soft MD and KG MD models. This work is organized as follows: in section 2 we review the structure of the Rouse model and the ideas of Silberberg relating to the conformations of polymer chains in melt at a surface. These ideas motivate a modification of the Rouse model to include surface effects. We shall be interested primarily in the behaviour of the resulting model for chains consisting of a large number of bonds, analogous to the continuous Rouse model, which may be compared with the behaviour of reasonably long but non-entangled KG MD chains. Generally, the modified-Rouse model is solved numerically, although the longest relaxation behaviour of a surface-tethered chain is accurately modelled using a dumbbell, tethered by one end to a reflective surface. We derive an analytic expression for the end-to-end vector correlation function of the dumbbell in the wall normal direction. We show that the functional form of the dumbbell end-to-end vector correlation function closely matches the first Rouse mode auto-correlation function of a chain consisting of many bonds, and that the correspondence between the two functions matches that of the standard analytic Rouse model, applicable to 
chains without boundary constraints.

In section 3, the modified-Rouse model is compared to KG MD, via the end-to-end vector and individual Rouse mode (mode numbers $p>0$ ) correlation functions of surface-tethered chains. We investigate chain lengths spanning the non-entangled regime (comprising 32, 64 or 128 bonds). Whilst we find that the choice of thermostat friction constant has little effect on the end-to-end correlation function, which is dominated by the longest Rouse modes, Farago et al ${ }^{29,30}$ have shown that the zeroth Rouse mode, i.e the centre of mass motion, deviates strongly from simple diffusion at times shorter than the Rouse time, and is strongly dependent on the coupling between chain elasticity and hydrodynamics. In section 4 we show that the surface parallel component of the zeroth mode motion displays enhanced visco-elastic hydrodynamic coupling in the presence of a perfectly smooth flat surface. Final conclusions are made in section 5 .

\section{The Rouse model with a reflective boundary condition}

A simple class of single-chain model assumes that the dynamics of a polymer chain, consisting of $N+1$ connected beads, may be described via a set of $N+1$ Langevin equations, which without external force are of form: ${ }^{27,37}$

$$
\frac{d \mathbf{R}_{i}}{d t}=\sum_{j=0}^{N} \mathbf{H}_{i j}\left(-\frac{\partial U}{\partial \mathbf{R}_{j}}+\mathbf{f}_{j}^{r}(t)\right)+\frac{T}{2} \sum_{j=0}^{N} \frac{\partial}{\partial \mathbf{R}_{j}} \mathbf{H}_{i j}
$$

for $i=0,1, \ldots, N$. The position vector of bead $i$ is denoted by $\mathbf{R}_{i}$. In this work we consider such a model of a single polymer chain in a channel bounded in only one direction such that $y_{w 1}<y_{i}<y_{w 2}$, where the positions of the channel walls are denoted by $y_{w 1}$ and $y_{w 2}$; we

resolve $\mathbf{R}_{i}$ into surface normal and surface parallel components, $\mathbf{R}_{i}=\mathbf{R}_{i, \|}+y_{i} \hat{\mathbf{e}}_{\perp}$, where $\hat{\mathbf{e}}_{\perp}$ is the wall normal direction unit vector.

In the case of the Rouse model, the assumption that hydrodynamic interactions are screened 
leads to a simple form for the mobility tensor:

$$
\mathbf{H}_{i j}=\frac{\delta_{i j} \mathbf{I}}{\xi}
$$

where I denotes the three-dimensional unit matrix. This assumption is usually considered valid for the melt or concentrated solution. $\mathbf{f}_{i}^{r}(t)$ is then a Gaussian white-noise random variable:

$$
\begin{aligned}
& \left\langle\mathbf{f}_{i}^{r}(t)\right\rangle=0 ; \\
& \left\langle\mathbf{f}_{i}^{r}(t) \mathbf{f}_{j}^{r}\left(t^{\prime}\right)\right\rangle=2 T \xi \delta\left(t-t^{\prime}\right) \delta_{i j} \mathbf{I}
\end{aligned}
$$

where $\xi$ is the bead friction constant, and $T$ is the temperature. Throughout this work temperature is taken in units of the Boltzmann constant, $k_{B}$. Eqn (1) has the irreversible characteristic that if we begin with an ensemble of systems with arbitrary bead positions, as $t \rightarrow \infty$, the ensemble probability distribution reaches equilibrium, where the equilibrium probability of a given set of bead positions specifying a system state is related to the potential $U$ as: ${ }^{27}$

$$
p\left(\left\{\mathbf{R}^{N+1}\right\}\right)=\frac{\exp \left(-\frac{U\left(\left\{\mathbf{R}^{N+1}\right\}\right)}{T}\right)}{Z_{1}}
$$

where $Z_{1}$ is the ensemble partition function. $\left\{\mathbf{R}^{N+1}\right\}$ indicates the set of all bead coordinates. Assuming that sub-segments of polymer chains satisfy Gaussian statistics on all length scales, from eqn (4) Rouse found a harmonic potential acting between beads:

$$
U_{\text {Rouse }}=\sum_{i=1}^{N} \frac{k}{2}\left(\mathbf{R}_{i}-\mathbf{R}_{i-1}\right)^{2}
$$

where $k=3 T / b^{2}$ is the spring constant. $b$ is the characteristic length of the Gaussian chain segment. Each chain segment has mean square length $b^{2}$.

In this work we investigate whether the assumptions of the Rouse model are equally accurate 
near the surface. Namely that:

1. Hydrodynamic interactions are screened: beads experience isotropic friction, eqn (2).

2. Time dependent topological constraints are not considered.

Under these assumptions, eqns (1), (2) and (3) remain valid, but with a modified potential $U$, corresponding to a different probability distribution of segment orientations. We determine the modified potential by requiring that the equilibrium probability distribution corresponds to a random walk with a particular boundary condition. Crucially, any such model reduces to the Rouse model far from surfaces. In 1982 Silberberg advocated that the reflective boundary condition is appropriate to describe melt statistics at a flat surface. ${ }^{38}$

\section{Silberberg's hypothesis - surface chain conformations are reflected random walks}

Silberberg's hypothesis is an extension of the Flory hypothesis ${ }^{24}$ for polymer melts at a flat surface. As a result of the effective incompressibility of the melt state, the monomer density remains almost uniform up to an interface. Silberberg argued that since monomers experience an isotropic environment right up to the surface, Flory's argument should hold equally well in this region. Then the probability distribution for the orientation of a chain bond near (but not in direct contact with) a flat impenetrable surface is identical to the bulk distribution, having no preferred direction.

In the simplified case of a random walk lattice chain model, the hypothesis means that locally the polymer melt at a flat surface looks the same as the unconfined melt. The only difference being that steps beginning at the surface are constrained to move away from it, therefore satisfying reflective boundary conditions. Silberberg showed that a method of images like conformation swap procedure conserves the unconfined segment partition function and respects the boundary constraint. Since the random walk uncouples in directions parallel and normal to the surface, the conformation swap only affects the wall normal probability distribution. 
Sussman et $\mathrm{al}^{8}$ have noted that the conformation swap procedure of Silberberg may be generalized to the case of chains bounded by two surfaces in a channel of width $L$. The total partition function (number of walks) of a chain with fixed start position inside the channel is conserved irrespective of the channel width, as must be the case under reflective boundary conditions. If the $i$ th segment is near to one of the surfaces, and we allow the channel width to go arbitrarily large, $L \rightarrow \infty$, the probability distribution for the wall normal end position of the segment, $y_{i}$, under the conditions of segment start position, $y_{i-1}$, and wall position, $y_{w}$, reduces to the form found by Silberberg, given by a sum of two unbounded walk weights (in the continuous limit):

$$
\begin{aligned}
& P\left(y_{i} \mid y_{i-1}, y_{w}\right)=P\left(y_{i} \mid y_{i-1}\right)+P\left(\bar{y}_{i} \mid y_{i-1}\right)= \\
& \frac{1}{\sqrt{2 \pi \sigma_{1}^{2}}} \exp \left(-\frac{\left(y_{i}-y_{i-1}\right)^{2}}{2 \sigma_{1}^{2}}\right) \\
& +\frac{1}{\sqrt{2 \pi \sigma_{1}^{2}}} \exp \left(-\frac{\left(\bar{y}_{i}-y_{i-1}\right)^{2}}{2 \sigma_{1}^{2}}\right)
\end{aligned}
$$

where $\bar{y}_{i}$ is the surface reflected end position $\bar{y}_{i}=y_{w}-\left(y_{i}-y_{w}\right)$, and $\sigma_{1}^{2}=b^{2} / 3$. In the supplementary material we find eqn (43) directly by solving the formal boundary value problem with a pair of reflective boundaries in the limit $L \rightarrow \infty$.

\section{Modified-Rouse potential}

We propose a potential for eqn (1) when a chain segment is close to a flat impenetrable surface in a wide channel, using eqn (43) and the general relation, eqn (4). The final potential comprises the Rouse potential, eqn (5), and an additional term due to the surface which depends on the set, $\left\{y^{N+1}\right\}$, of bead positions in the wall normal direction, relative to the surface position:

$$
U\left(\left\{\mathbf{R}^{N+1}\right\}\right)=U_{\text {Rouse }}\left(\left\{\mathbf{R}^{N+1}\right\}\right)+\sum_{i=1}^{N} A\left(y_{i}, y_{i-1}, y_{w}\right)
$$


where the additional term is:

$$
A\left(y_{i}, y_{i-1}, y_{w}\right)=-T \ln \left(1+\exp \left(-\frac{2\left(y_{i}-y_{w}\right)\left(y_{i-1}-y_{w}\right)}{\sigma_{1}^{2}}\right)\right)
$$

Woo et al $^{39}$ similarly derived a potential using absorbing boundary conditions. Absorbing boundaries are considered appropriate for an ideal polymer chain in dilute solution. We refer the reader to the supplementary material for a derivation of the resulting potential in both cases.

If the channel width, $L$, is very large compared to the characteristic chain segment length, $b$, the contribution to the partition function corresponding to segment conformations which are reflected at both surfaces is negligible. This is certainly the case for the weakly confined simulations that we study in this work; therefore we neglect the finite channel corrections ${ }^{8}$ to eqn (43) and use a system potential consisting of two wall interaction terms of the form given in eqn (8), corresponding to the two surfaces.

\section{Single chain (modified-Rouse) model numerical scheme}

Using the potential given by eqn (7), adapted to a system with two parallel walls, we solve the set of equations (1), (2) and (3) numerically, using a two-stage predictor-corrector algorithm for the conservative force, the simplest Euler scheme for the random force ${ }^{37}$ and reflective boundary conditions. The algorithm is:

$$
\begin{aligned}
& \overline{\mathbf{R}}_{i}(t+\Delta t)=\mathbf{R}_{i}(t)+\frac{\Delta t}{\xi} \mathbf{f}_{i}^{c}(\mathbf{R}(t))+\sqrt{\frac{2 T \Delta t}{\xi}} \mathbf{n}_{t} \\
& \mathbf{R}_{i}(t+\Delta t)=\overline{\mathbf{R}}_{i}(t+\Delta t)+\frac{\Delta t}{2 \xi}\left(\mathbf{f}_{i}^{c}(\overline{\mathbf{R}}(t+\Delta t))-\mathbf{f}_{i}^{c}(\mathbf{R}(t))\right)
\end{aligned}
$$

where $\mathbf{f}_{i}^{c}(\mathbf{R}(t))=-\left.\frac{\partial U\left(\left\{\mathbf{R}^{N+1}\right\}\right)}{\partial \mathbf{R}_{i}}\right|_{t}$ is the conservative force, and $\mathbf{n}_{t}$ is a normally distributed random vector with unit variance in each direction. If the particle crosses a boundary its position is reflected in the boundary plane. We are aware of numerical errors in this 
scheme and more advanced algorithms have been proposed. ${ }^{40}$ For the present case, we have confirmed convergence of the numerical scheme, and validated agreement with static and dynamic analytic results where available. In the bulk, the natural length unit is $b$. We find the natural time unit, $\tau_{b}$, from the bead diffusion for very small $t$ :

$$
\tau_{b}=\frac{\xi b^{2}}{6 T}
$$

Accurate results are found using a time-step $\Delta t=\tau_{b} / 10$. Smaller time-steps yield nearly identical results.

\section{Mapping to the single-chain (modified-Rouse) model}

While a number of detailed studies on coarse-graining interacting many-chain systems exist ${ }^{41}$ much less is known about mapping a many-chain system to an effective single-chain model. ${ }^{42}$ In order to investigate the usefulness of the modified-Rouse model for describing polymer dynamics of non-entangled melts near surfaces, we study two multi-chain beadspring 'fundamental' models as references; both described in more detail in section 3.

We restrict ourselves to fundamental systems with chains containing an even number, $n$, of bonds, such that we can define a mapping ratio $m: 1$ to the modified-Rouse model comprising $N=n / m$ bonds and $N+1$ beads, where $m$ is a divisor of $n$. The only constraint within our mapping is that we pick $b$ such that the chain mean square end-to-end distance matches the fundamental model in the bulk:

$$
b=\sqrt{\frac{\left\langle\mathbf{u}_{\text {bulk }}^{2}\right\rangle}{N}}
$$

where $\left\langle\mathbf{u}_{\text {bulk }}^{2}\right\rangle$ is the mean square end-to-end distance of bulk chains within the fundamental model using periodic boundaries. Then the degree to which other system observables, such as more general internal square distances, are successfully mapped, depends upon the accuracy of reflected random walk statistics on all length scales. Results presented in section 3 will 
test the extent of this agreement. Within the modified-Rouse model we keep $T=1$. This leaves two unknown parameters in the model: the mapping ratio $m$ which decides $N$, and the friction coefficient $\xi$. These two parameters are chosen from the best simultaneous fit of the auto-correlation functions of the longest Rouse modes of surface-tethered chains. For a more systematic derivation of single-chain friction coefficients using projection operator techniques, see e.g. Akkermans et $\mathrm{al}^{43}$ and Ilg et al. ${ }^{44}$

Before mapping the fundamental models, we first investigate the general dynamical behaviour of chains end-tethered at the surface using the modified-Rouse model.

\section{Dynamics of the modified-Rouse model}

For a chain connected by harmonic bonds, the dynamic equations of the Rouse normal coordinates (modes) uncouple for all possible chain conformations. However, for such a chain experiencing confinement, the real space description of dynamics cannot be replaced by uncoupled Rouse modes, since they are effectively coupled via the boundary conditions. We are left then with a complicated system of coupled equations and boundary conditions even without the additional potential, eqn (8). Despite this, the Rouse mode correlation functions remain important general observables, since they describe the decay of chain structure correlations with characteristic segment number 'wavelength' $m_{p} \approx \frac{2 N}{p}$, where $p$ is the mode number. All correlation functions which depend solely on chain bond orientations can be written as a function of Rouse mode auto- and cross-correlation functions. Should the mapped modified-Rouse model predict these correlation functions well, all orientation correlation functions must be well described.

In a different approach to Vladkov et al, ${ }^{14}$ in which Rouse mode analysis was performed over a limited time duration on free chains binned from chain positions in the channel at $t=0$, we first investigate the dynamics of chains which have a constraint keeping them in the surface region for all time. We choose to make the constraint by fixing one of the end beads at the surface. Surface-adsorbed chains themselves strongly influence rheological properties. ${ }^{45,46}$ 
We analyse the orientational relaxation of the surface-tethered chains in terms of the normal modes of unbounded Rouse chains with one end fixed. These coordinates differ slightly from those usually referred to by 'Rouse modes' (this term is usually reserved for the normal modes of Rouse chains with free ends), and are derived for finite $N$ in A. Likhtman's short course: 'Entangled Polymer Dynamics': 47

$$
\mathbf{X}_{p}=\frac{1}{N+1 / 2} \sum_{i=1}^{N} \mathbf{R}_{i} \sin \left(\frac{\pi i(p-1 / 2)}{N+1 / 2}\right)
$$

For simplicity we refer to the set of coordinates, $\mathbf{X}_{p}$, as 'Rouse modes', to emphasize that they correspond to the normal modes of tethered chains within the Rouse model.

We now show that the functional form of the longest Rouse mode correlation function within a modified-Rouse model of a surface-tethered chain can be accurately modelled by an analytic dumbbell model.

\section{End-to-end correlation function of a surface-tethered dumbbell}

The dynamics of the modified-Rouse model uncouple in Cartesian coordinates providing that one axis coincides with the surface normal direction. Since the dynamical equations in each parallel direction coincide, we define dynamic functions for directions normal, $\perp$, and parallel, $\|$, to the surface. In this work, when we label functions using the subscripts $\perp$ and $\|$, this always refers to functions of surface-tethered chains unless otherwise stated.

The end-to-end vector correlation function is an experimentally accessible observable for type-A dipolar polymers, being proportional to the correlation function of the chain electric dipole in this case. For end-tethered chains, in the $\alpha$ direction it may be generally written in terms of the Rouse coordinates, eqn (12), as: ${ }^{47}$

$$
\left\langle u_{\alpha}(t) u_{\alpha}(0)\right\rangle=4 \sum_{p, q=1}^{N}(-1)^{p+q} \cos \left(\frac{\pi(p-1 / 2)}{2(N+1 / 2)}\right) \cos \left(\frac{\pi(q-1 / 2)}{2(N+1 / 2)}\right)\left\langle X_{p, \alpha}(t) X_{q, \alpha}(0)\right\rangle
$$


where $u_{\alpha}$ are components of the end-to-end vector. We define a normalized end-to-end vector correlation function with respect to the mean in each direction:

$$
\Phi_{\alpha}(t)=\frac{\left\langle\left(u_{\alpha}(t)-\left\langle u_{\alpha}\right\rangle\right)\left(u_{\alpha}(0)-\left\langle u_{\alpha}\right\rangle\right)\right\rangle}{\left\langle u_{\alpha}^{2}\right\rangle-\left\langle u_{\alpha}\right\rangle^{2}}
$$

For a dumbbell, the normalized end-to-end correlation function, $\Phi_{\mathrm{db}, \alpha}(t)$, coincides with the first normalized Rouse mode auto-correlation function, $A_{1, \alpha}(t)$. The $A_{p, \alpha}(t)$ are:

$$
A_{p, \alpha}(t)=\frac{\left\langle\left(X_{p, \alpha}(t)-\left\langle X_{p, \alpha}\right\rangle\right)\left(X_{p, \alpha}(0)-\left\langle X_{p, \alpha}\right\rangle\right)\right\rangle}{\left\langle X_{p, \alpha}^{2}\right\rangle-\left\langle X_{p, \alpha}\right\rangle^{2}}
$$

Within the modified-Rouse model for an end-tethered chain, the surface normal component of the end-to-end vector is described by a half Gaussian distribution where the first two moments are:

$$
\begin{aligned}
& \left\langle u_{\perp}\right\rangle=\sqrt{2\left\langle u_{\perp}^{2}\right\rangle / \pi} \\
& \left\langle u_{\perp}^{2}\right\rangle=\left\langle u_{\|}^{2}\right\rangle=N b^{2} / 3
\end{aligned}
$$

where $u_{\|}$is a component of the end-to-end vector in a surface parallel direction. Resultantly, in the case of a surface-tethered dumbbell, the corresponding potential has a simple harmonic form and therefore corresponds to an Ornstein-Uhlenbeck process ${ }^{27,37}$ with a reflective boundary at the potential minimum. For details of the analytic solution of the end-to-end vector correlation function of this dumbbell model, $\Phi_{\mathrm{db}, \perp}(t)$, we refer the reader to the supplementary material. The final expression is:

$$
\Phi_{\mathrm{db}, \perp}(t)=\frac{1}{\pi / 2-1}\left(\arctan \left(\mathrm{e}^{-\frac{t}{\tau_{\mathrm{db}, \|}}} \frac{1}{\sqrt{1-\mathrm{e}^{-\frac{2 t}{\tau_{\mathrm{db},}, \|}}}}\right) \mathrm{e}^{-\frac{t}{\tau_{\mathrm{db}, \|}}}+\sqrt{1-\mathrm{e}^{-\frac{2 t}{\tau_{\mathrm{db}, \|}}}}-1\right)
$$

where $\tau_{\mathrm{db}, \|}$ is the relaxation time of $\Phi_{\mathrm{db}, \|}(t)$ which is simply the standard Ornstein-Uhlenbeck process position correlation function. 


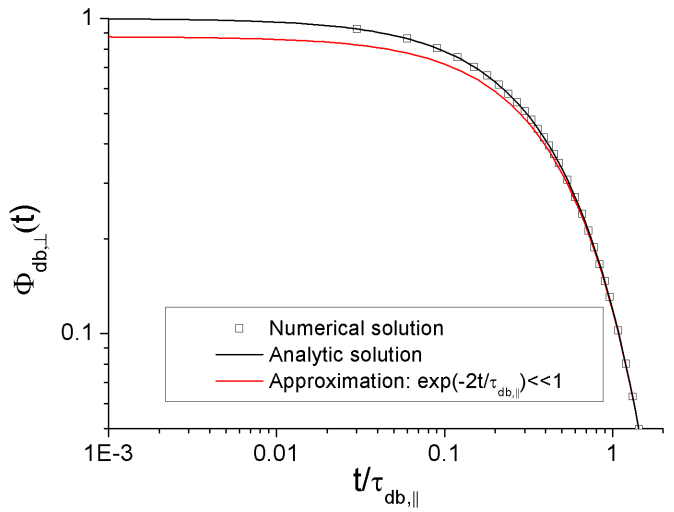

Figure 1: Comparison of $\Phi_{\mathrm{db}, \perp}(t)$, eqn (63), with its long time asymptotic expansion, eqn (19), and numerical solution.

\section{Regimes of $\Phi_{\mathrm{db}, \perp}(t)$}

Expanding the dumbbell end-to-end vector correlation function to leading order around $t=0$ gives:

$$
\begin{aligned}
& \left\langle\left(u_{\perp, \mathrm{db}}(t)-\left\langle u_{\perp, \mathrm{db}}\right\rangle\right)\left(u_{\perp, \mathrm{db}}(0)-\left\langle u_{\perp, \mathrm{db}}\right\rangle\right)\right\rangle \approx \frac{b^{2}}{3}\left(1-t / \tau_{\mathrm{db}, \|}\right)-\frac{2 b^{2}}{3 \pi} \\
& \approx\left\langle u_{\|, \mathrm{db}}(t) u_{\|, \mathrm{db}}(0)\right\rangle-\left\langle u_{\perp, \mathrm{db}}\right\rangle^{2}
\end{aligned}
$$

so that in the limit $t / \tau_{\mathrm{db}, \|} \rightarrow 0\left(\Phi_{\mathrm{db}, \perp}(t)\right.$ is not analytic at $\left.t=0\right)$, the derivative of the dumbbell end-to-end vector correlation function in the wall normal direction is identical to the parallel direction. The dumbbell relaxes as if it were in the unbounded direction, since it has not yet interacted with the surface.

For $\exp \left(-2 t / \tau_{\mathrm{db}, \|}\right) \ll 1$, expanding eqn $(63)$ in $\epsilon=\exp \left(-2 t / \tau_{\mathrm{db}, \|}\right)$ to leading order gives:

$$
\Phi_{\mathrm{db}, \perp}(t) \approx \frac{1}{\pi-2} \exp \left(-2 t / \tau_{\mathrm{db}, \|}\right)
$$

In figure 1, eqn (19) is compared to the exact form, eqn (63), revealing that for $t \gtrsim \tau_{\mathrm{db}, \|} / 2$, $\Phi_{\mathrm{db}, \perp}(t)$ is well approximated by a single exponential with relaxation time $\tau_{\mathrm{db}, \|} / 2$. In the intermediate regime between eqn (18) and eqn (19), there is a stronger decay, well described 
by a power-law, $\Phi_{\mathrm{db}, \perp}(t) \propto 1 / \sqrt{t}$.

In addition, figure 1 compares the analytic and numeric solutions for $\Phi_{\mathrm{db}, \perp}(t)$. This presents one validation of our numerical Brownian dynamics method.

Correspondence between $\Phi_{\mathrm{db}, \perp}(t)$ and $A_{1, \perp}(t)$ (for $N \rightarrow \infty$ )

For the modified-Rouse model the Rouse modes completely uncouple parallel to the surface, so that the functions $A_{p, \|}(t)$ remain single exponential irrespective of $N{ }^{47}$ Since for a dumbbell we have: $\Phi_{\mathrm{db}, \alpha}(t)=A_{1, \alpha}(t)$, for $N \rightarrow \infty, A_{1, \|}(t)$ matches $\Phi_{\mathrm{db}, \|}(t)$ exactly providing we use the correct correspondence between the bead friction coefficient in each model: $\xi \rightarrow \xi_{\mathrm{db}}$ (In addition to matching the mean square end-to-end distance of the dumbbell and the chain) where $\xi_{\mathrm{db}}$ is the corresponding free bead dumbbell friction. This correspondence is found from equating the longest relaxation times of the models: $\tau_{1, \|}=\tau_{\mathrm{db}, \|}$. For an unbounded end-tethered Rouse chain, the expression for the mode relaxation times are: ${ }^{47}$

$$
\tau_{p, \|}=\frac{\xi b^{2}}{12 T \sin ^{2}\left(\frac{\pi(p-1 / 2)}{2(N+1 / 2)}\right)}
$$

For $N \gg 1$, the longest relaxation time $\tau_{1, \|}$ is well approximated by its limit $\tau_{R, \|}(N \rightarrow \infty)$ :

$$
\tau_{R, \|}=\frac{4 \xi N^{2} b^{2}}{3 \pi^{2} T}
$$

Equating $\tau_{R, \|}$ with the corresponding relaxation time for a fixed dumbbell gives the limiting relation between $\xi$ and $\xi_{\mathrm{db}}$ :

$$
\xi_{\mathrm{db}}=\frac{4 \xi N}{\pi^{2}}
$$

Then if the same correspondence applies in the wall normal direction, the asymptotic ratio of longest relaxation times in directions normal and parallel to the surface, must match the 


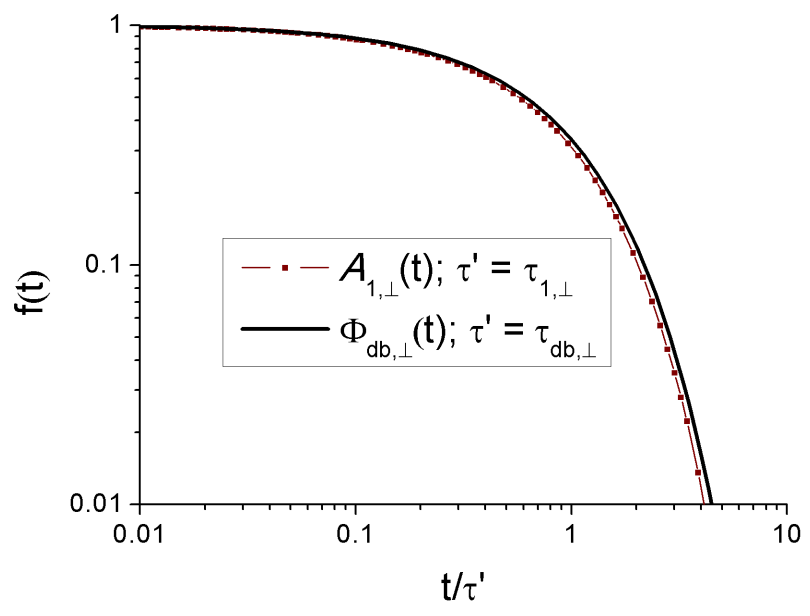

Figure 2: Comparison of the analytic solution of $\Phi_{\mathrm{db}, \perp}(t)$, eqn $(63)$, with $A_{1, \perp}(t)$ using a chain of 32 bonds within the modified-Rouse model. We assume $\tau_{1, \perp}=\tau_{1, \|} / 2$.

dumbbell ratio which we already know:

$$
\frac{\tau_{R, \perp}}{\tau_{R, \|}}=\frac{\tau_{\mathrm{db}, \perp}}{\tau_{\mathrm{db}, \|}}=\frac{1}{2}
$$

Figure 2 compares $A_{1, \perp}(t)$ for a modified-Rouse chain comprising 32 bonds with the analytic solution of $\Phi_{\mathrm{db}, \perp}(t)$ (eqn (63)). The time axis is normalized assuming the relationship eqn (65), where $\tau_{1, \|}$ is given by eqn (64). As for the parallel direction, described by standard Rouse theory, the large $N$ converged behaviour of $A_{1, \perp}(t)$ is reached by $N \approx 32$. In the supplementary material, we explore the convergence of $\Phi_{\perp}(t)$ as more bonds are used to model a chain.

Figure 2 implies that the asymptotic relation, eqn (22), remains appropriate in the wall normal direction, and that the form of $A_{1, \perp}(t)$ is very close to being conserved from a dumbbell to a chain consisting of many bonds. Specifically, we see the same power-law crossover from the initial decay, eqn (18), to the final single exponential behaviour, eqn (19), with relaxation time very close to half that of the unbounded case.

For intermediate modes we see the same qualitative behaviour: for modes $p=2,3, A_{p, \perp}(t)$ (plotted and compared with the KG MD model in section 3) may be fitted to the form of the dumbbell end-to-end correlation function reasonably well, although at very long times 
there are stronger deviations from the single-exponential form. The faster modes mostly decay without interacting with the surface, and therefore we see a return to the unbounded behaviour for $A_{p, \perp}(t) ; p \gg 1$. Resultantly, the mode relaxation times in the wall normal direction approach those of the wall parallel direction for $p \gg 1$. From eqn (64), taking $N \rightarrow \infty$, the relationship between successive mode relaxation times in the parallel direction becomes:

$$
\frac{\tau_{p, \|}}{\tau_{p+1, \|}}=\frac{(p+1 / 2)^{2}}{(p-1 / 2)^{2}}
$$

In figure 3 we compare the first six Rouse relaxation times in the wall parallel direction, $\tau_{p, \|}$, found from eqns (21) and (24), with the corresponding relaxation times in the normal direction, $\tau_{p, \perp}$, which are found by fitting the functions $A_{p, \perp}(t)$ to a single exponential using the Reptate software. ${ }^{48}$ The relaxation times, $\tau_{p, \perp}$, are found using a modified-Rouse model consisting of 256 bonds. For this chain bond number and $p<7, \tau_{p, \|}$ has effectively asymptotic behaviour: eqn (24). Figure 3 shows that already at $p=4, \tau_{p, \perp} / \tau_{p, \|}>0.9$.

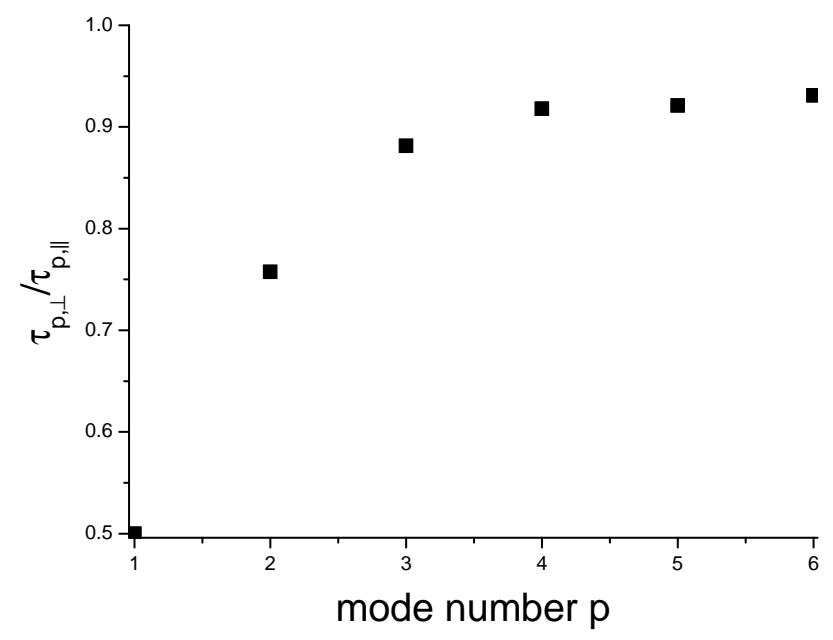

Figure 3: The ratio, $\tau_{p, \perp} / \tau_{p, \|}$, of relaxation times of the first six Rouse mode correlation functions for a chain end-tethered to the surface $(N=256)$, using the modified-Rouse model.

In the supplementary material, the behaviour of the Rouse mode cross-correlation functions are investigated. Contrary to the unbounded Rouse model, cross-correlations do not vanish in the modified normal direction. Although non-negligible, the influence of cross-correlations 
is small. Resultantly $A_{1, \perp}(t)$ still dominates the terminal behaviour of $\Phi_{\perp}(t)$.

\section{Comparing the modified-Rouse model to multi-chain mod-}

\section{els}

The purpose of the following two sections is to compare the modified-Rouse model with interacting multi-chain models which are coarse-grained descriptions that nevertheless exhibit the most important features of flexible polymer melts at the time and length scales of interest. The primary model we will use is the well-studied KG MD model. ${ }^{16}$ This model has the realistic characteristic that bonds may not cross one another. We also investigate a second model, soft MD, which comprises soft bonded and non-bonded potentials, with parameters chosen such that, in the bulk, the mean square internal distances along a chain,

$\left\langle\left(\mathbf{r}_{i}-\mathbf{r}_{j}\right)^{2}\right\rangle /|i-j|$, match almost exactly those of KG MD; whilst simultaneously allowing bond crossing events. Making a comparison between these two models may help to elucidate the importance of chain topology in dynamics. The soft MD model has previously been used to examine the effect of entanglements on chain orientation coupling, ${ }^{49}$ and as a means for faster equilibration of KG MD systems; ${ }^{50}$ details of soft MD and the parameters we use are given in these references. All MD simulations with confinement use periodic boundaries in the $\hat{\mathbf{x}}$ and $\hat{\mathbf{z}}$ directions and reflective boundaries in the $\hat{\mathbf{y}}$ direction, whereby particle positions and velocities are reflected if they pass the surface plane. The box dimensions in the periodic directions were set as $L_{z}, L_{x} \approx 2 \sqrt{\left\langle\mathbf{u}_{\text {bulk }}^{2}\right\rangle}$. In order to test against finite periodic box dimension effects, some simulations were doubled in size by cloning all polymer chains and displacing the cloned chains by $L_{x}$, then resetting the box dimension in the $\hat{\mathbf{x}}$ direction to $2 L_{x}$. After re-equilibration, the larger systems showed no significant deviations from the original ones in typical static or dynamic observables. Bulk simulations use periodic boundaries in all directions.

The KG MD model consists of Lennard-Jones (LJ) beads connected via a FENE bonded 
potential:

$$
\begin{aligned}
& U_{\mathrm{LJ}}(r)=4 \epsilon\left((\sigma / r)^{12}-(\sigma / r)^{6}+1 / 4\right), \quad r / \sigma<2^{1 / 6} \\
& U_{\mathrm{FENE}}(r)=-\frac{K R_{0}^{2}}{2} \ln \left(1-\left(r / R_{0}\right)^{2}\right)
\end{aligned}
$$

where the FENE parameters are $R_{0}=1.5 \sigma$ (corresponding to the maximum bond extension) and $K=30 \epsilon / \sigma^{2}$ (spring constant). $\sigma$ is the LJ bead radius and $\epsilon$ is the LJ energy. The shortest natural time unit is set by the Lennard-Jones potential: $\tau_{\mathrm{LJ}}=\sqrt{\sigma^{2} m_{\mathrm{b}} / \epsilon}$, where $m_{\mathrm{b}}$ is the bead mass. The LJ potential we use is purely repulsive, corresponding to a cut-off at $2^{1 / 6} \sigma$. The temperature is maintained via a Langevin thermostat. In all plots, unless otherwise stated, we use a thermostat friction constant $\xi_{\mathrm{MD}}=0.5 m_{b} / \tau_{\mathrm{LJ}}$ at $T=\epsilon$. This is the most common value for the friction constant in the literature. ${ }^{16,18,51-53}$ Under these parameters the maximum time-step implemented is $\Delta t=0.012 \tau_{\mathrm{LJ}}$ using periodic boundaries and $\Delta t=0.01 \tau_{\mathrm{LJ}}$ using reflecting boundaries. Correlation functions generated using a smaller time-step produce negligibly different results. We also present some results using a much weaker friction constant, $\xi_{\mathrm{MD}}=0.05 m_{b} / \tau_{\mathrm{LJ}}$, in order to explore the influence of momentum conservation on the dynamics. For the lower friction constant we use $\Delta t=0.002 \tau_{\mathrm{LJ}}$. We have verified that a uniform temperature profile is maintained across the channel under these conditions. Little difference in dynamical behaviour is found if $\xi_{\mathrm{MD}}$ is reduced beyond $\xi_{\mathrm{MD}}=0.05 m_{b} / \tau_{\mathrm{LJ}}$ for all observables considered. For the chain lengths of interest, the larger, most standard friction constant, $\xi_{\mathrm{MD}}=0.5 m_{b} / \tau_{\mathrm{LJ}}$, is itself an intermediate value for which there remain non-negligible effects due to inertia. Our comparison between the high and low friction cases is therefore not a comprehensive study of the influence of momentum conservation on the chain dynamics near surfaces, but should be sufficient to observe the main trends. Importantly, for soft MD the dimensionless friction constant we use is close to the high friction KG MD value.

The equations of motion are solved using the Verlet algorithm. ${ }^{54}$ We calculate all correlation functions using the multiple-tau correlator code developed by Ramirez et al. ${ }^{55}$ For the mean 
square displacement observable the block averaging is not performed, in order to avoid all systematic error.

\section{Molecular dynamics equilibration procedure}

Within this work, all chain lengths (except $n=128$ ) are below the threshold at which entanglement effects become important in the bulk, ${ }^{16,36}$ which greatly reduces the time of equilibration. Free chains are initially placed in the simulation box with random start positions. The other beads are then grown with mean equilibrium bond length and bond angles that correspond closely to the equilibrium distribution. Tethered chain orientations are similarly generated, but the start beads are ordered into a periodic face-centred cubic lattice in the (111) plane. An example system snapshot including tethered chains is given in figure 4. The interaction potentials are gradually turned on ('push-off') over a single Lennard-Jones
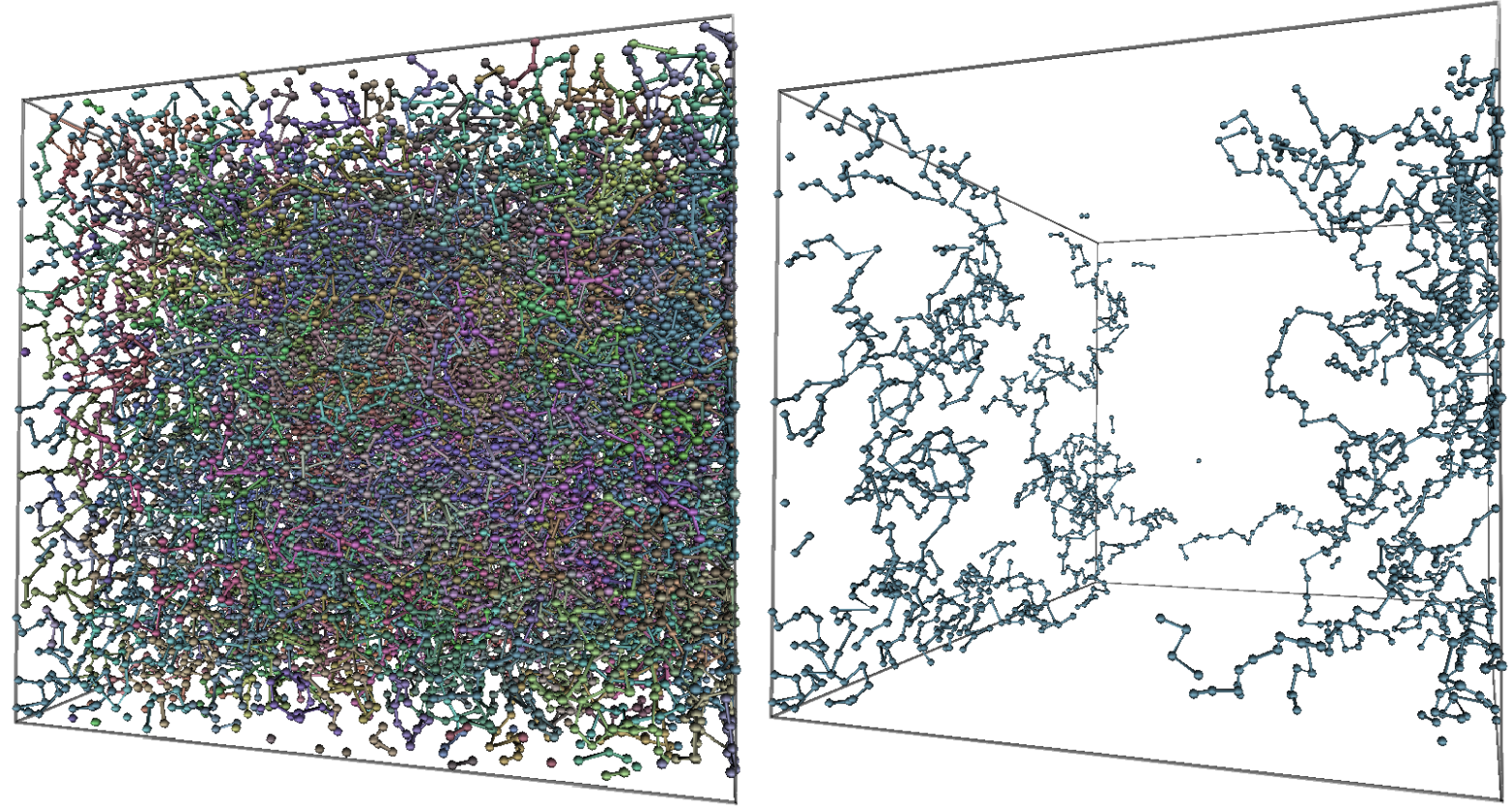

Figure 4: A typical simulation box snapshot for the surface-tethered system. Left: all chains included. Right: only surface-tethered chains shown.

time unit. ${ }^{53}$ The boundary condition leads to a small increase in density at the surface relative to the channel centre. The initial volume is estimated such that the density in the 
middle of the channel is close to the melt density, $\rho=0.85 \sigma^{-3}$, that we use for simulations with periodic boundaries in all directions. Then, during a second equilibration step, the simulation box is affinely compressed or extended very slowly until the middle channel density reaches $0.85 \pm 0.001 \sigma^{-3}$. This equilibration step is typically quick, since the initially chosen density is itself very close to the desired value. The simulation then runs for several longest system relaxation times before any observations are made. The mean square internal distances are also monitored as a means to check that the systems are well equilibrated.

\section{Mean square internal distances of reflected random walks}

Sarabadani et al ${ }^{18}$ have already compared the static behaviour of an almost identical KG MD variant to the one we use, with reflected random walks; including the cases of attractive and

repulsive surface interactions. Their results were in agreement with those of Skvortsov et al, ${ }^{56}$ who compared the Self Consistent Field (SCF) calculations of a wall tethered chain immersed in a melt at different wall-fluid interaction strengths. The results confirmed that static properties of a melt at a surface are largely unaffected by changes in boundary interactions. All cases universally agreed well with a single-chain under critical conditions, of which there are analytic expressions for bead density profiles. ${ }^{57}$ The MD simulations did however reveal some deviations from the Silberberg picture: the entropic surface interaction of chain ends ${ }^{58}$ is not described, and the mean field description cannot take into account the surface induced ordering of monomers. ${ }^{17}$

We now present some additional static results for the multi-chain models, which show that the dominant deviations from reflected random walk statistics at the surface are similar to known deviations from random walk statistics in the bulk. ${ }^{25}$

In figure 5 we plot the wall normal component of the mean square end-to-end distance of chain sub-segments consisting of $m$ bonds, such that at least one end be a distance $y$ from the surface, which we refer to as $\left\langle u_{m, \perp}^{2}(y)\right\rangle$. For reflected random walks, the internal distance 
profile is: ${ }^{38}$

$$
\frac{\left\langle u_{m, \perp}^{2}(y)\right\rangle}{\left\langle u_{m, \perp}^{2}(\infty)\right\rangle}=1-(4 / \sqrt{\pi}) \nu \exp \left(-\nu^{2}\right)+4 \nu^{2}(1-\operatorname{erf}(\nu))
$$

where $\nu=y / \sqrt{2 \sigma_{m}^{2}}$ is a characteristic dimensionless distance and $\sigma_{m}^{2}=\left(m b^{2}\right) / 3$. Figure $\left.5 \mathrm{~d}\right)$ shows that if we map KG MD and soft MD multi-chain models to a reflected random walk, such that the mean square end-to-end distances match in the bulk, they also match closely across the whole channel. Agreement between soft MD and KG MD for the chain internal distances remains good at all points in the channel and at all length scales $\left(\left\langle u_{m, \perp}^{2}(y)\right\rangle\right.$ for $m=8,16,32$ are plotted in figures 5 (a), (b), (c) respectively). The most notable difference between the two models is that soft MD has smaller wall normal mean square end-to-end distances for segments with ends very close to the surface.

Across the channel, the mapped reflected random walks tend to predict larger mean square internal distances than are found for sub-segments of multi-chain models, since segments are progressively more swollen as the bond number increases. However, at around $\nu=1$, the reflected random walk end-to-end distances approach, and actually become slightly smaller than the corresponding multi-chain model values. That upon approaching the surface the internal distances of the reflected random walks decrease at a faster rate compared to the real chain segments they are mapped from, can be understood easily; they are more extended and therefore more likely to come into contact with the surface, reducing the end-to-end distance. The oscillations that are apparent for short segments are associated with the wall ordering phenomenon influenced by our choice of boundary condition. ${ }^{17}$ We have performed additional simulations using lattices of Lennard-Jones beads acting as an explicit boundary. Increasing the roughness of the lattice reduces surface ordering of monomers. At the density in question, $\rho=0.85 \sigma^{-3}$, we find that the degree of bead ordering has no significant effect on chain dynamics near to the surface for all observables considered here.

Sarabadani et $\mathrm{al}^{18}$ have also shown that surface chains become swollen in the surface parallel direction. We find that in the surface parallel direction the mean square end-to-end distance of complete KG MD chains comprising $n=64$ bonds is around $10 \%$ larger for chains be- 

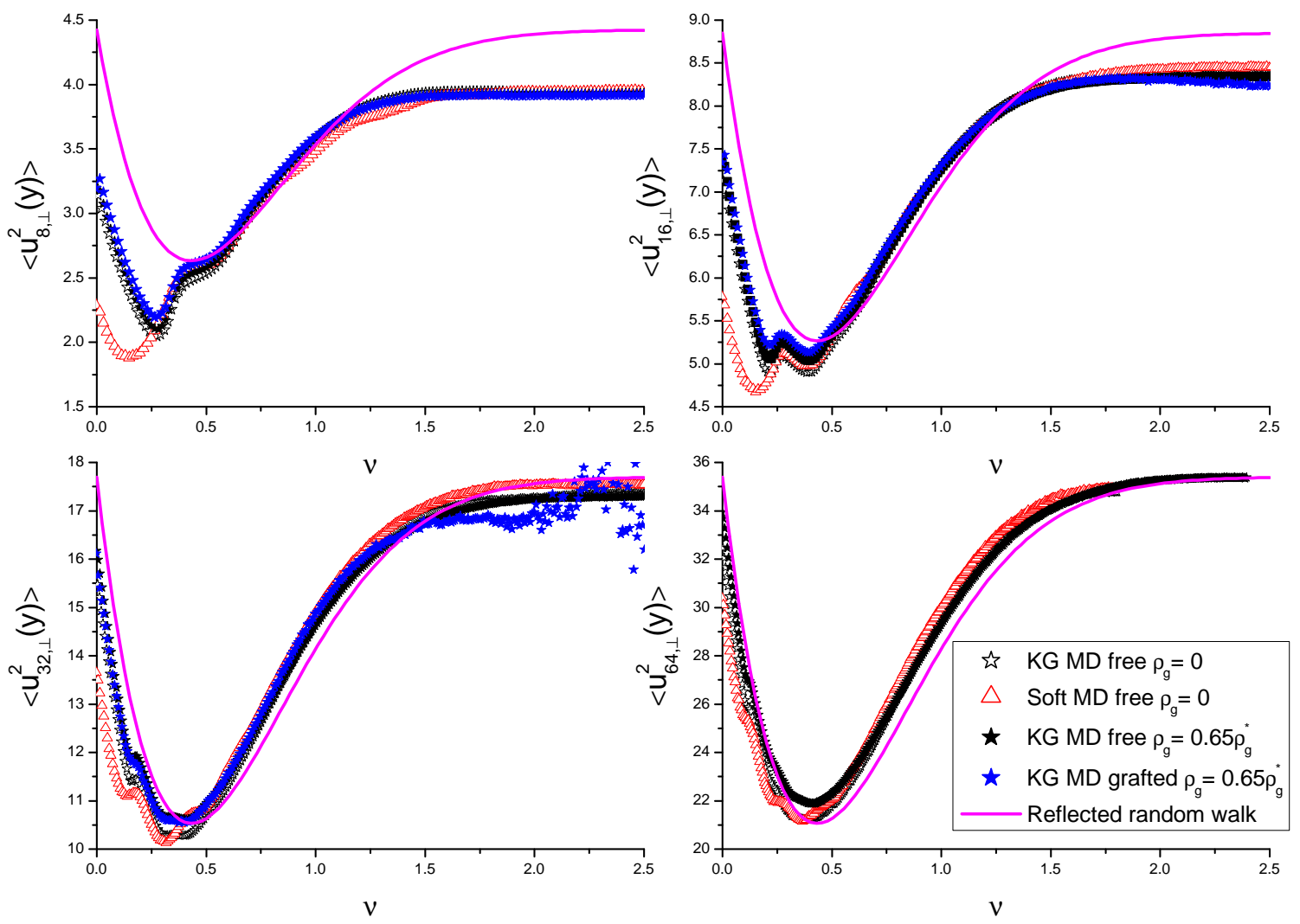

Figure 5: Comparison of KG MD with the mapped reflected random-walk prediction, eqn (25), for mean square internal distances, $\left\langle u_{m, \perp}^{2}(y)\right\rangle$, as a function of the reduced distance $\nu=y / \sqrt{2 \sigma_{m}^{2}}$, where $y$ is the distance from an end bead to the surface, for sub-chains consisting of $m$ bonds. a) $m=8$, b) $m=16$, c) $m=32$, d) $m=64$. The chains consist of $n=64$ bonds. 
ginning with one end at the surface, compared to bulk chains, as shown in figure 6 . The

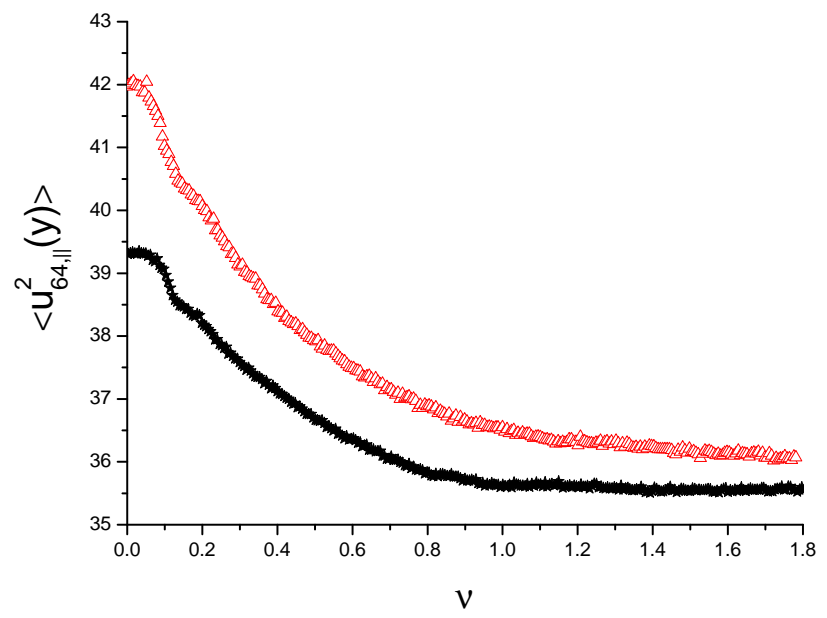

Figure 6: Complete chain mean square end-to-end distance in the surface parallel direction for chains with one end at distance $y$ from the surface. $\nu=y / \sqrt{2 \sigma_{64}^{2}}$. Red: soft MD, black: KG MD. The chains consist of $n=64$ bonds.

chains do not appear swollen in the perpendicular direction (figure 5); in fact, the end-toend dimension is slightly shrunk with respect to the reflected random walk prediction. This suggests that the surface induces a weak nematic chain alignment which we investigate in the supplementary material. For soft MD chains the corresponding swelling and contraction effect is slightly more pronounced.

We are also interested in systems with identical chain properties and architecture but which are subject to constraints. These are our tethered chain systems, whose micro-states coincide with a sub-ensemble of the confined free-chain system; whereby there are chains with an end-bead fixed at regular lattice sites on the reflective surface. Figure 5 ((a), (b) and (c)) includes a comparison of $\left\langle u_{m, \perp}^{2}(y)\right\rangle$ for free and tethered chains, within a system at surface grafting density of end-tethered chains, $\rho_{g}=0.65 \rho_{g}^{*}$, where we define an approximate 'overlap density', $\rho_{g}^{*}$, which gives correct scaling for the grafting density at which neighbouring tethered chains begin to interact and stretch:

$$
\rho_{g}^{*}=\frac{1}{\left\langle\mathbf{u}_{\text {bulk }}^{2}\right\rangle}
$$


The grafting density we use is sufficiently low such that the square internal distances of both the tethered and free chains almost exactly coincide with the completely free chain system. We therefore expect very similar dynamical behaviour of tethered chains compared to free chains with one end bead at the surface.

The free chain system used a smaller channel height of $3 \sqrt{\left\langle\mathbf{u}_{\text {bulk }}^{2}\right\rangle}$, compared to $4 \sqrt{\left\langle\mathbf{u}_{\text {bulk }}^{2}\right\rangle}$ for the tethered system. This increases computational speed and only leads to a very small difference in the mean square end-to-end distance of the complete chain in the middle of the channel (figure $5 \mathrm{~d}$ )). For channel heights smaller than $3 \sqrt{\left\langle\mathbf{u}_{\text {bulk }}^{2}\right\rangle}$, there is no bulk region and the surface chain behaviour begins to deviate from weak confinement.

\section{Rouse mode correlation functions of surface-tethered chains - map- ping from KG MD}

We map to the modified-Rouse model from surface-tethered chains immersed in a free chain matrix using the KG MD system. Both free and tethered chains consist of the same number, $n$, of bonds. The two free parameters, $m$ and $\xi$, are determined by ensuring the best simultaneous agreement between the models for the first four Rouse mode correlation functions, $A_{p, \perp}(t) ; p=1,2,3,4$, of the tethered chains, which are plotted in figure 7 a). For $n=64$, at grafting density $\rho_{g}=0.65 \rho_{g}^{*}$, we find $m=2$ and $\xi / m=22 m_{\mathrm{b}} / \tau_{\mathrm{LJ}}$, where the mapping ratio is $1: m$. Using this mapping, $A_{1, \perp}(t)$ is very well matched between the models. As the mode number increases, the corresponding correlation functions in the KG MD system gain stronger stretched exponential behaviour, but the dominant contribution is universally well described by the modified-Rouse model. In figure 7 b) we see the same behaviour for free chains in periodic boundaries, using correlation functions of the Rouse modes of free chains, and the same mapping parameters as for the fixed chains. Figure $7 \mathrm{~b}$ ) includes plots for the low thermostat friction, $\xi_{\mathrm{MD}}=0.05 m_{b} / \tau_{\mathrm{LJ}}$, with the time axis corresponding to these plots given in reduced units, $\tau_{\text {reduced }}=0.86 \tau_{\mathrm{LJ}}$, which takes into account the simulation speed-up resulting from the change in friction constant. With this rescaling of time we see close agree- 

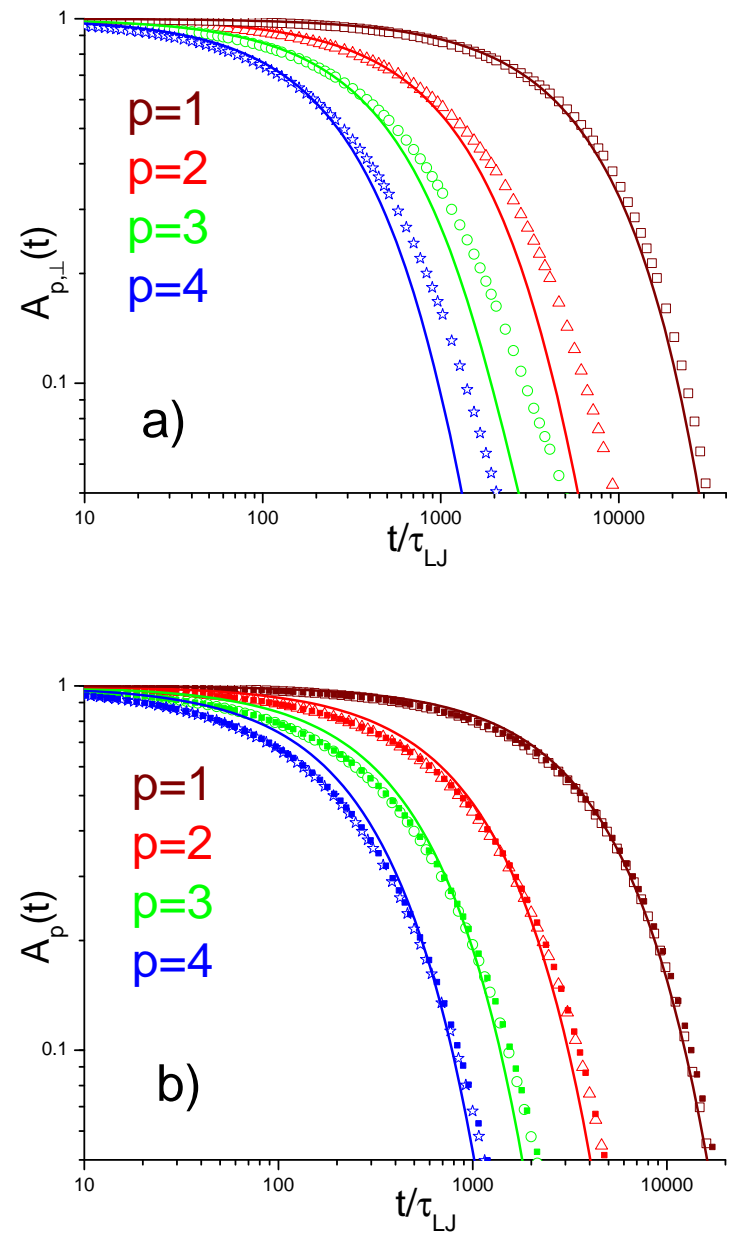

Figure 7: For chains consisting of $n=64$ bonds and mode number $p=1,2,3,4$ from right to left. Open symbols and lines correspond to the KG MD model and mapped modified-Rouse model respectively. a) First four Rouse mode correlation functions, $A_{p, \perp}(t)$, eqn (15), in the surface normal direction, for surface-tethered chains at grafting density $\rho_{g}=0.65 \rho_{g}^{*}$. b) First four Rouse mode correlation functions of free chains, using periodic boundaries for the KG MD model, compared to the analytic Rouse model. Filled squares correspond to $\mathrm{KG}$ MD using the reduced friction, $\xi_{\mathrm{MD}}=0.05 \mathrm{~m}_{b} / \tau_{\mathrm{LJ}}$, plotted in reduced time units, $\tau_{\text {reduced }}=0.86 \tau_{\mathrm{LJ}}$. 


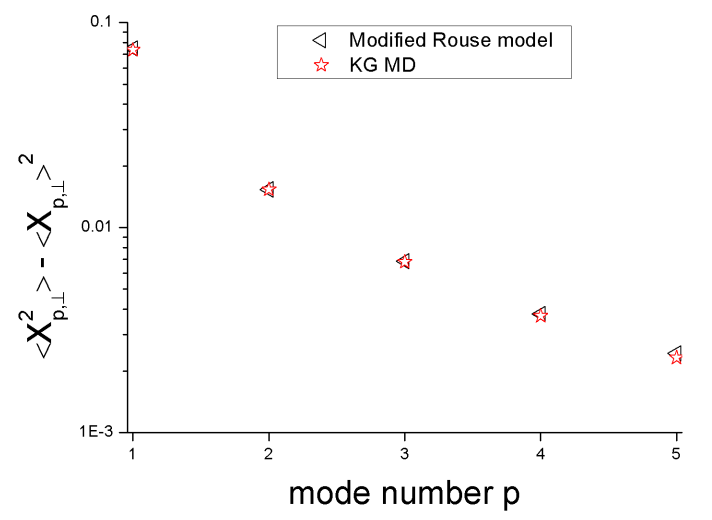

Figure 8: Surface normal amplitudes (variance) of Rouse modes for surface-tethered KG MD chains at grafting density $\rho_{g}=0.65 \rho_{g}^{*}$, compared to the mapped reflected random walk (modified-Rouse) prediction using mapping ratio $2: 1$. The amplitudes are normalized by the bulk chain mean square end-to-end distance in one direction. $n=64$.

ment for all Rouse modes using both high and low friction constants. However, the zeroth mode describing the centre of mass diffusion is more strongly dependent on the thermostat friction $^{30}$ (see Section 4 for further discussion).

Figure 8 shows that the good agreement with reflected random walk statistics on large scales corresponds with good agreement for the Rouse mode amplitudes at small $p$. Resultantly, since the small $p$ modes (particularly the first mode) dominate $\Phi_{\alpha}(t)$ (and Rouse mode crosscorrelations are also small for KG MD), we get excellent agreement between the models for $\Phi_{\perp}(t)$. For $p \gg 4$ the modified-Rouse model fails to predict $A_{p, \perp}(t)$ accurately within the KG MD model using this mapping. We see that $\Phi_{\perp}(t)$ (figure 9 a)), described by the modifiedRouse model, predicts the corresponding KG MD correlation functions as accurately as the analytic Rouse model does for $\Phi_{\|}(t)$ (Figure 9 b)) and the end-to-end vector correlation function of free chains in the bulk (figure 9 c)) using periodic boundaries. In figure 9 a) and b), plots corresponding to the low friction system in units of $\tau_{\text {reduced }}$ are given for $n=32$, which match the high friction plots well. We find that Rouse mode correlation functions of tethered chains are also well matched between the high and low friction cases for $n=32$, upon renormalization of time-scales using $\tau_{\text {reduced }}=0.86$ (not plotted). In figure 9 we use a bond mapping ratio of $2: 1$ up to a maximum of $N=32$. The friction coefficients used are: 
for $n=32: \xi / m=20 m_{\mathrm{b}} / \tau_{\mathrm{LJ}} ;$ for $n=64,100,128,200: \xi / m=22 m_{\mathrm{b}} / \tau_{\mathrm{LJ}}$. In all cases, the same friction coefficients and mapping ratios are used in both the periodic and bounded simulations, meaning that the modified-Rouse model can simultaneously give a good description of both bulk and surface chains. That we also use the same friction coefficients in parallel and normal directions means that the theoretical prediction for the ratio of relaxation times, eqn (65), is valid within the KG MD system.

The plots corresponding to tethered chains consisting of $n=32$ or 64 bonds have a grafting density $\rho_{g}=0.65 \rho_{g}^{*}$. The plots for $n=128$ used a higher grafting density, $\rho_{g}=1.3 \rho_{g}^{*}$; however, chain stretching due to 'brush' interactions remains minimal. We therefore associate the deviations from the single-chain model prediction at $n=128$ bonds with the onset of entanglements, which may be compared with the analogous deviations of free chains comprising $n=100$ and 200 bonds, shown in figure 9 c).

\section{Effect of the flat boundary condition on viscoelastic-hydrodynamic}

\section{coupling in polymer melts}

In simulation, a widely used observable is the mid-bead mean square displacement $g_{1, \operatorname{mid}}(t)$. For a chain consisting of an even number of bonds, $g_{1, \text { mid }}(t)$ can be written in a particularly simple form in terms of the normal coordinates of free Rouse chains: ${ }^{36}$

$$
g_{1, \text { mid }}(t)=\sum_{p, q=0, \text { even }}^{N}(-1)^{(p+q) / 2}\left(2-\delta_{p 0}\right)\left(2-\delta_{q 0}\right)\left\langle\left(\mathbf{X}_{p}(t)-\mathbf{X}_{p}(0)\right)\left(\mathbf{X}_{q}(t)-\mathbf{X}_{q}(0)\right)\right\rangle
$$

$g_{1, \text { mid }}(t)$ generally encompasses motion of the chain on all length scales, unlike the end-toend vector correlation function, eqn (13). In the Rouse model, cross-correlations do not 

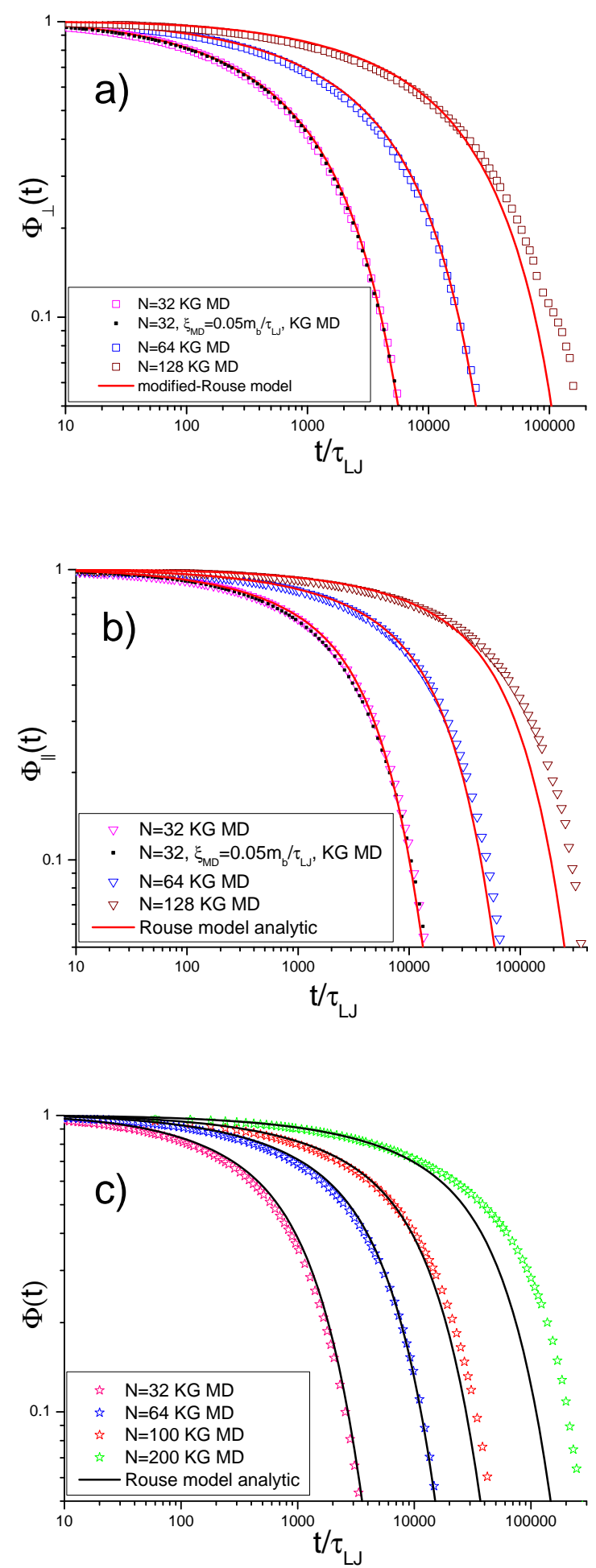

Figure 9: Normalized end-to-end vector correlation function in directions normal to, a), and parallel to, b), the surface, for surface-tethered KG MD chains mapped to the modifiedRouse model and analytic Rouse respectively. The tethered chains consist of $n=32,64$, or 128 bonds. For $n=32, \mathrm{KG}$ MD using reduced friction, $\xi_{\mathrm{MD}}=0.05 m_{b} / \tau_{\mathrm{LJ}}$, is plotted in reduced time units, $\tau_{\text {reduced }}=0.86 \tau_{\mathrm{LJ}}$. c) End-to-end vector correlation function for free bulk chain systems consisting of $n=32,64,180$ or 200 bonds, mapped to the analytic Rouse prediction. 
contribute, and eqn (27) may be written as:

$$
g_{1 \text { Rouse } \text { mid }}(t)=\frac{6 T t}{(N+1) \xi}+\frac{b^{2}}{(N+1)} \sum_{p=2, \text { even }}^{N} \frac{\left[1-\exp \left(-\frac{t}{\tau_{p}}\right)\right]}{\sin ^{2}\left(\frac{\pi p}{2(N+1)}\right)}
$$

The first term is the centre of mass mean square displacement:

$$
g_{3 \text { Rouse }}(t)=\frac{6 T t}{(N+1) \xi}
$$

Eqn (29) results immediately for any stochastic model only comprising bonded forces. Upon a transformation to the centre of mass coordinate, all forces are pairwise and cancel. This leaves a sum of uncorrelated Wiener processes acting on the $N+1$ beads in the chain. Even in the bulk, $g_{3}(t)$ of real chains is not diffusive on short time scales. Both experiment and simulation find a sub-diffusive regime, which for $t<\tau_{R}$ has characteristic scaling $g_{3}(t) \propto t^{\nu_{3}}$, $\nu_{3} \approx 0.8 .^{32}$ This sub-diffusive regime has been attributed to hydrodynamic-viscoelastic couplings, ${ }^{29,30}$ and soft sphere like interactions between nearest neighbour chains. ${ }^{32}$ In figure 10 we plot the centre of mass diffusion of free chains in both wall parallel, $g_{3, \|}(t) /\left(t / \tau_{R}\right)$, and normal, $g_{3, \perp}(t) /\left(t / \tau_{R}\right)$, directions, for chains with different start positions relative to the surface in a channel of width $3 \sqrt{\left\langle\mathbf{u}_{\text {bulk }}^{2}\right\rangle}$. Chains are assigned to narrow bins of wall normal width $0.25 \sqrt{\left\langle\mathbf{u}_{\text {bulk }}^{2}\right\rangle}$ at $t=0$, depending on the distance, $y_{\mathrm{cm}}$, from the centre of mass to the nearest wall at that time. For example, the bin closest to the surface corresponds to chains that at $t=0$ have centre of mass position located within a distance $0.25 \sqrt{\left\langle\mathbf{u}_{\text {bulk }}^{2}\right\rangle}$ from the surface. Then the correlation functions are calculated for a duration $\approx 1.7 \tau_{R}$. In this time the chains move a distance normal to the surface of approximately the bin diameter. For soft MD, the Rouse time is found from the centre of mass mean square displacement in the diffusive limit, using a simulation box with periodic boundaries, assuming the relation, eqn (29); whereas for KG MD the Rouse time is found from our previously fitted friction value, $\xi / 2=22 m_{b} / \tau_{\mathrm{LJ}}$ (this also applies to mid-bead mean square displacement in the next 
sub-section). For each model, the simulation was repeated more than a hundred times. The resulting statistics are good enough to ensure that in a corresponding simulation of the modified Rouse model, the relative error in the final correlation point for the mid-bead mean square displacement observable with respect to the analytic Rouse expression is less than 1\%. Again we use chains consisting of $n=64$ bonds within the fundamental models, and mapping ratio $2: 1$. We also include plots corresponding to systems using periodic boundaries which are in good agreement with similar simulations from an existing study, ${ }^{30}$ whose theory predicts an enhanced super-diffusive regime at early time followed by the subdiffusive regime until $t \approx \tau_{R}$. We find that for KG MD chains beginning with centre of mass in the channel centre, for $g_{3, \|} /\left(t / \tau_{R}\right)$, figure $\left.10 \mathrm{a}\right)$, the results match closely to the periodic simulations except that the sub-diffusive regime has a weaker slope. Moving closer to the surface a stronger hydrodynamic interaction (HI) becomes apparent in the parallel direction through a stronger super-diffusive regime for both high and low thermostat friction cases. Although in the channel centre it appears that the high and low friction cases tend to the same diffusive limit, which matches closely to the periodic simulation, it appears that this is not the case closer to the surface. It is clear that the final diffusion coefficient appears faster closer to the surface. This is in contrast to the parallel centre of mass diffusion using the soft MD model, figure $10 \mathrm{~b}$ ). In this case all regions appear to tend to the same diffusive limit, although, at early time, the increase in the super-diffusion near to the surface is similar to KG MD $(\approx 10 \%)$. However it is only for the two bins closest to the surface that deviations from the channel centre behaviour become discernible. It is interesting to note that the sub-diffusive decay of the diffusion constant for Soft MD using periodic boundaries scales with $t^{-0.12}$, whereas for KG MD the scaling follows $t^{-0.2}$ : see dashed lines in figure 10 .

We have also performed preliminary simulations investigating $g_{3}(t)$ using boundaries formed from Lennard-Jones beads with varying degrees of roughness. We find that in the limit of zero roughness the results tend to the flat reflective surface case. Increasing the roughness reduces the strength of the super-diffusive regime near the surface, and thus appears to 
counteract hydrodynamic effects, such that at sufficiently large surface roughness the early time parallel centre of mass diffusion is actually reduced near the surface compared to the channel centre. At an intermediate surface roughness we find that the HI effect is close to constant across the channel so that $g_{3, \|}(t)$ is almost independent of channel position. We find that $g_{3, \perp}(t)$ is close to independent of surface roughness.

The comparison of KG MD with Soft MD may suggest that to some degree the increased rate of diffusion occurring for KG MD surface chains is associated with a decrease in topological interactions at the surface. However it must be noted that the viscoelastic-hydrodynamic effect could behave differently for soft MD, and we have not sufficiently investigated this in order to unambiguously associate the qualitative differences between KG MD and Soft MD with the topological interaction, independently of hydrodynamics.

In the surface normal direction, figure $10 \mathrm{c}$ ), we find that both high and low friction cases appear to tend to the same diffusive limit and that in contrast to the surface parallel direction there appears to be little difference in the strength of HI effects closer to the surface. Interestingly the channel centre diffusion in the normal direction appears to tend to a higher diffusive limit compared to periodic simulations (We observe the same feature using soft MD - not plotted).

It is clear that at the surface, as in the bulk, the simple centre of mass diffusion on all time scales predicted by the Rouse model parallel to the surface, eqn (29), is strongly inaccurate.

\section{Chain mid-monomer mean square displacement}

The deviations from Rouse behaviour due to hydrodynamic-viscoelastic couplings are not

expected to be as strong for individual bead diffusion, ${ }^{30}$ which is instead dominated by the bonded forces and expected to follow the Rouse scaling more closely. For $\tau_{N}<t<\tau_{R}$, 

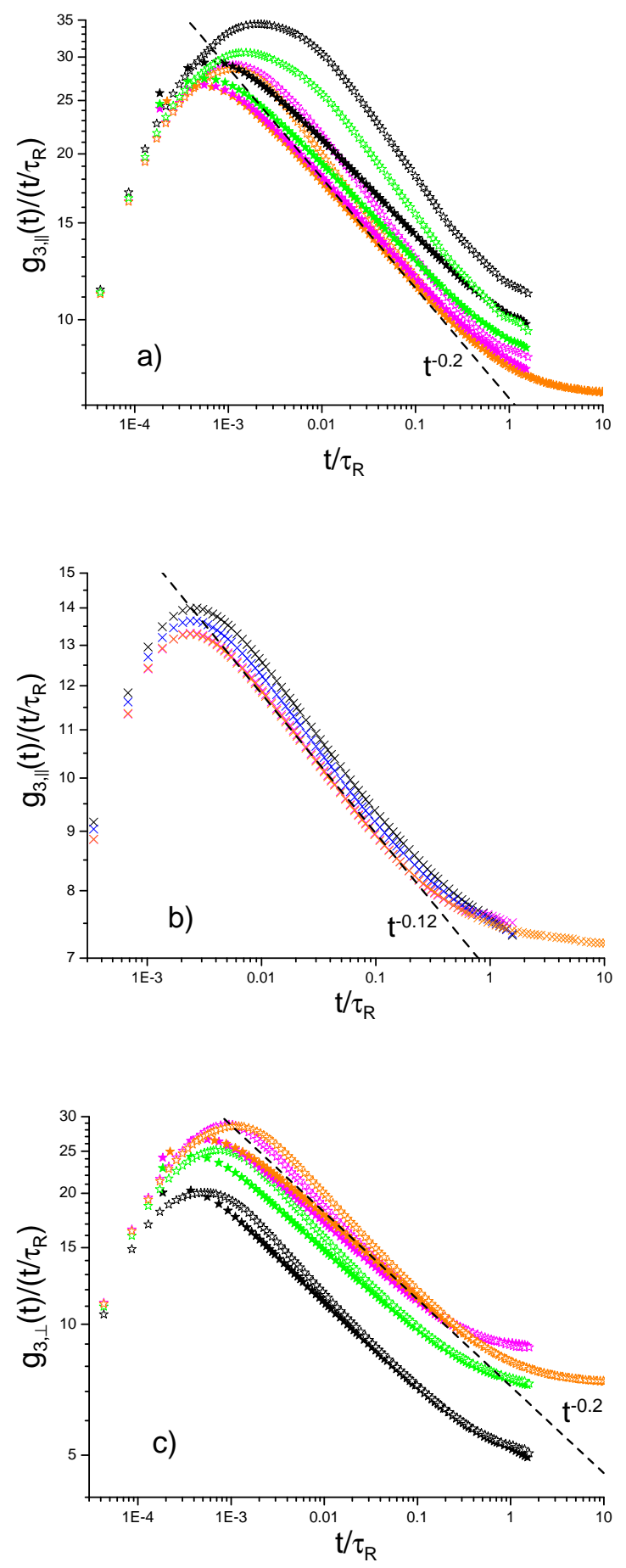

Figure 10: Centre of mass time dependent diffusion rate in the wall parallel direction (a) KG MD, b) Soft MD) and wall normal direction (c) KG MD). Black: $y_{\mathrm{cm}}<0.25 \sqrt{\left\langle\mathbf{u}_{\text {bulk }}^{2}\right\rangle}$; blue: $0.25 \sqrt{\left\langle\mathbf{u}_{\text {bulk }}^{2}\right\rangle}<y_{\mathrm{cm}}<0.5 \sqrt{\left\langle\mathbf{u}_{\text {bulk }}^{2}\right\rangle}$; green: $0.5 \sqrt{\left\langle\mathbf{u}_{\text {bulk }}^{2}\right\rangle}<y_{\mathrm{cm}}<0.75 \sqrt{\left\langle\mathbf{u}_{\text {bulk }}^{2}\right\rangle} ;$ magenta: $1.25 \sqrt{\left\langle\mathbf{u}_{\mathrm{bulk}}^{2}\right\rangle}<y_{\mathrm{cm}}$; orange: periodic boundaries. Filled stars are KG MD using $\xi_{\mathrm{MD}}=$ $0.5 m_{b} / \tau_{\mathrm{LJ}}$ and open stars are KG MD using $\xi_{\mathrm{MD}}=0.05 m_{b} / \tau_{\mathrm{LJ}} . \tau_{R, \xi_{\mathrm{MD}}=0.05}=0.86 \tau_{R, \xi_{\mathrm{MD}}=0.5}$. 
$g_{1 \text { Rouse,mid }}(t)$ is well approximated by: ${ }^{59}$

$$
g_{1 \text { Rouse } \text { mid }}(t) \approx 2 b \sqrt{\frac{3 t T}{\pi \xi}} \approx \frac{2\left\langle\mathbf{u}^{2}\right\rangle}{\pi^{3 / 2}} \sqrt{\frac{t}{\tau_{R}}}
$$

where the second relation in eqn $(30)$ uses $N+1 \approx N$. The physical origin of the $\sqrt{t}$ scaling in eqn (30) is traced to an increasing effective drag that the bead experiences as its displacement becomes correlated with other beads along the chain. Here we repeat this argument as laid out by McLeish. ${ }^{59}$ Over time-scales in which a bead becomes aware of its connection to other beads in the chain, the sum of all forces acting along the correlated section of chain contour must balance on average, so that $g_{1}(t)$ only depends on the sum of uncorrelated random forces acting on the correlated beads. The mean number of other beads that a labelled bead is correlated with, $N_{\text {corr }}$, can be approximated in terms of its mean square displacement:

$$
N_{\text {corr }}(t)=\frac{g_{1}(t)}{b^{2}}
$$

Approximating $g_{1}(t)$ with the mean square displacement resulting from a sum of $N_{\text {corr }}$ un-

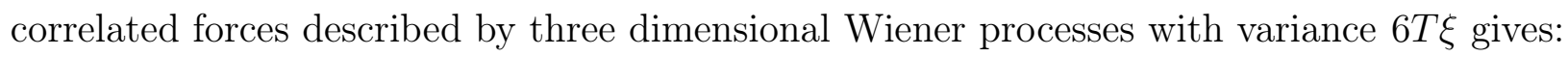

$$
g_{1}(t)=\frac{6 T t}{N_{\text {corr }}(t) \xi}=\sqrt{\frac{6 T b^{2} t}{\xi}}
$$

This matches eqn (30) up to the pre-factor. Since melt chains are not strictly ideal but experience swelling, if we wish to map the KG MD and soft MD models onto $N_{\text {corr }}(t)$ in eqn $(31), b^{2}$ becomes a time dependent increasing function. For this reason we can expect that for these models, at least in the bulk, $g_{1}(t) \propto t^{\nu_{1}}$ with $\nu_{1}>0.5$ for $\tau_{N}<t<\tau_{R}$.

Likhtman ${ }^{36}$ has compared the Rouse model prediction of $g_{1 \text {,mid }}(t)$ to soft MD and KG MD using periodic boundaries and free chains comprising $n=64$ bonds. Here we investigate the same observable for free chains confined in the channel of width $3 \sqrt{\left\langle\mathbf{u}_{\text {bulk }}^{2}\right\rangle}$.

Figure 11 includes plots of mid-bead mean square displacement in the wall normal di- 

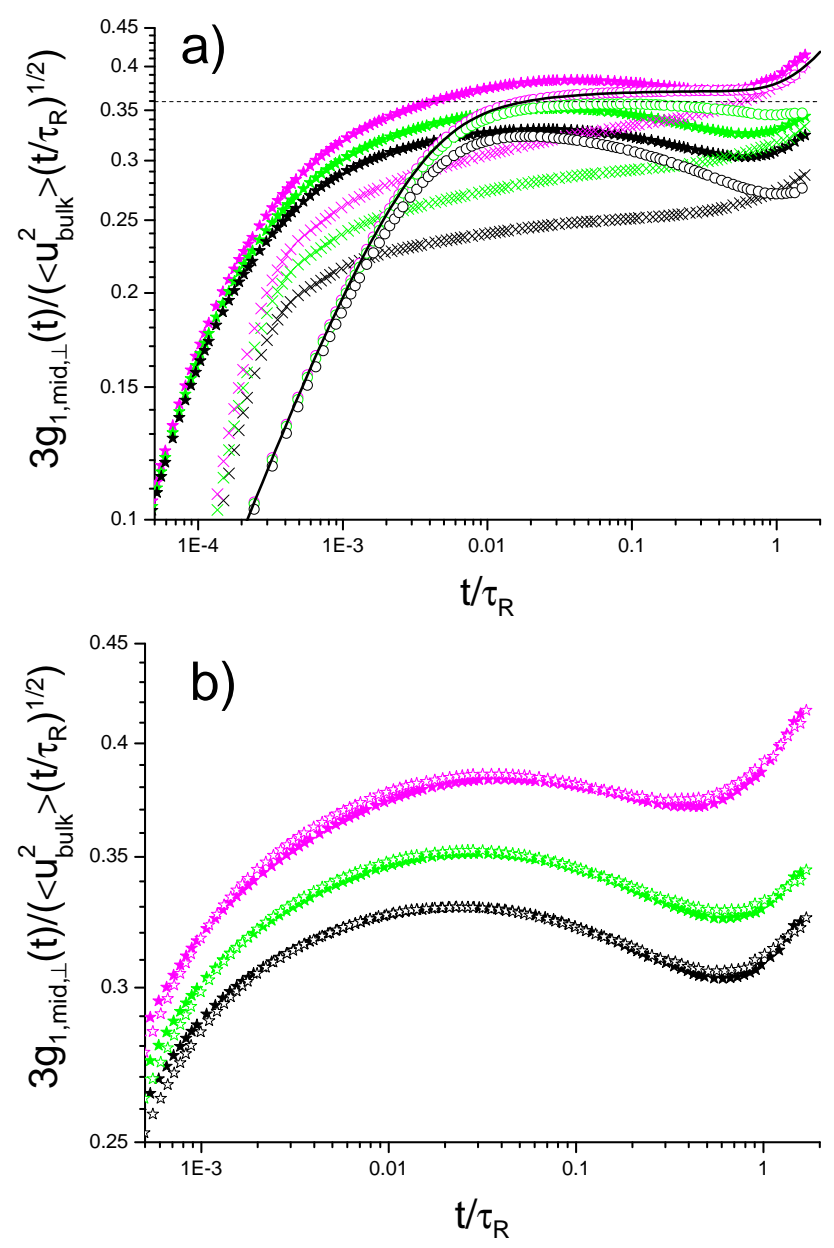

Figure 11: a) Mid-bead mean square displacement in the wall normal direction normalized to test the $\sqrt{t}$ scaling. Black: $y_{\text {mid }}<0.25 \sqrt{\left\langle\mathbf{u}_{\text {bulk }}^{2}\right\rangle} ;$ green: $0.5 \sqrt{\left\langle\mathbf{u}_{\text {bulk }}^{2}\right\rangle}<y_{\text {mid }}<0.75 \sqrt{\left\langle\mathbf{u}_{\text {bulk }}^{2}\right\rangle}$; magenta: $1.25 \sqrt{\left\langle\mathbf{u}_{\text {bulk }}^{2}\right\rangle}<y_{\text {mid }}$. Circles are modified-Rouse, crosses are soft MD, and filled stars are KG MD using $\xi_{\mathrm{MD}}=0.5 m_{b} / \tau_{\mathrm{LJ}}$; dashed line is $2 / \pi^{3 / 2}$ and solid black line is analytic Rouse. b) plots for KG MD using $\xi_{\mathrm{MD}}=0.5 m_{b} / \tau_{\mathrm{LJ}}$ are compared with KG MD using $\xi_{\mathrm{MD}}=0.05 m_{b} / \tau_{\mathrm{LJ}}$ (open stars). $\tau_{R, \xi_{\mathrm{MD}}=0.05}=0.86 \tau_{R, \xi_{\mathrm{MD}}=0.5}$. 


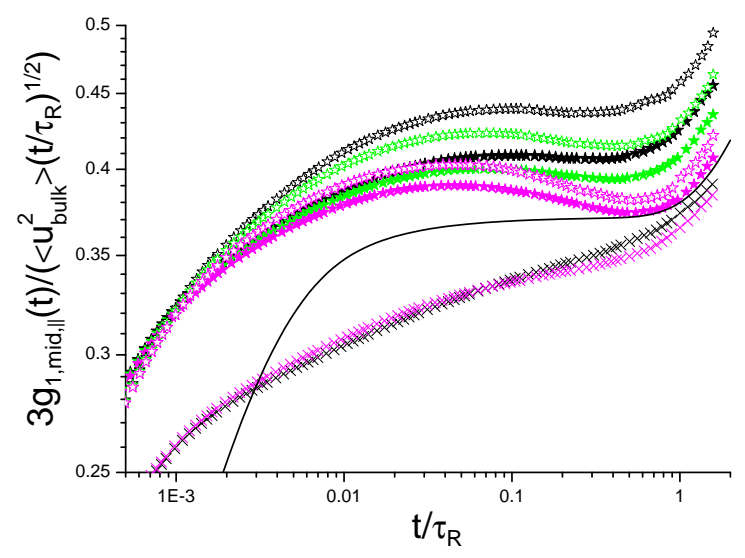

Figure 12: Mid-bead mean square displacement in the wall parallel direction normalized to test the $\sqrt{t}$ scaling. Black: $y_{\text {mid }}<0.25 \sqrt{\left\langle\mathbf{u}_{\text {bulk }}^{2}\right\rangle}$; green: $0.5 \sqrt{\left\langle\mathbf{u}_{\text {bulk }}^{2}\right\rangle}<y_{\text {mid }}<0.75 \sqrt{\left\langle\mathbf{u}_{\text {bulk }}^{2}\right\rangle}$; magenta: $1.25 \sqrt{\left\langle\mathbf{u}_{\text {bulk }}^{2}\right\rangle}<y_{\text {mid }}$. Solid black line is analytic Rouse, crosses are soft MD, filled stars and open stars are KG MD using $\xi_{\mathrm{MD}}=0.5 m_{b} / \tau_{\mathrm{LJ}}$ and $\xi_{\mathrm{MD}}=0.05 m_{b} / \tau_{\mathrm{LJ}}$ respectively. $\tau_{R, \xi_{\mathrm{MD}}=0.05}=0.86 \tau_{R, \xi_{\mathrm{MD}}=0.5}$.

rection $\left(g_{1, \text { mid, } \perp}\right)$, for chains with different start positions relative to the surface. For this observable the chains are assigned to bins at $t=0$ depending on the distance, $y_{\text {mid }}$, from the mid-bead to the nearest wall at that time. Then the correlation functions are again calculated for a duration $\approx 1.7 \tau_{R}$. The data is presented to test the scaling regime of eqn (30), normalized such that the Rouse model (in the limit $N \rightarrow \infty$ ) gives the value $2 / \pi^{3 / 2}$, for $\tau_{N} \lesssim t \lesssim \tau_{R}$. For $N=32$ the analytic Rouse solution has not yet met this limit, as apparent in figure 11 a). The analytic Rouse solution matches the modified-Rouse model in the centre of the channel, indicating that in this region the modified-Rouse chains are not influenced by the surface. The KG MD plot in figure 11 a) which represents chains satisfying $1.25 \sqrt{\left\langle\mathbf{u}_{\text {bulk }}^{2}\right\rangle}<y_{\text {mid }}$, matches closely to the Rouse plot for $t \gtrsim \tau_{R}$.

In figure 11 a) we observe that, before the first Rouse time is reached, $t<\tau_{N}, g_{1 \text {,mid }}(t)$ for the MD models is not captured well by modified-Rouse since Rouse has no super-diffusive regime. For $t \gtrsim 0.01 \tau_{R}$ the soft $\mathrm{MD}$ chains in the centre of the channel exhibit a scaling exponent $\nu_{1}>0.5$ as expected; however, the KG MD model matches the Rouse scaling more closely. This difference between KG MD and soft MD appears to suggest ${ }^{36}$ that adding chain 
topology slows down $g_{1, \text { mid }}(t)$ in such a way that the time-dependence of the speed-up due to the effect of chain swelling is counteracted.

Closer to the surface, for $0.5 \sqrt{\left\langle\mathbf{u}_{\text {bulk }}^{2}\right\rangle}<y_{\text {mid }}<0.75 \sqrt{\left\langle\mathbf{u}_{\text {bulk }}^{2}\right\rangle}$, all models are still in reasonable agreement at $t \approx \tau_{R}$; However, for chains beginning immediately at the surface, $y_{\text {mid }}<0.25 \sqrt{\left\langle\mathbf{u}_{\text {bulk }}^{2}\right\rangle}$, the values of the soft MD and modified-Rouse plots in figure 11 a) are less than $\mathrm{KG} \mathrm{MD}$, indicating that the relative drop in mobility from the channel centre to the surface is less for KG MD compared to the other two models. Analogous behaviour occurs for the surface parallel mid-bead mean square displacement shown in figure 12; for soft MD, the surface parallel diffusion is effectively constant across the channel, agreeing with the simple modified-Rouse model in this regard; whereas the KG MD model displays a weak increase in parallel mobility, and a slope which matches more closely to the Rouse plot for $t \gtrsim 0.1 \tau_{R}$, for chains near to the surface.

In figure 12 it is apparent that the $\mathrm{KG}$ MD model using the low thermostat friction has progressively faster surface parallel mid-bead diffusion for chains approaching the surface, with respect to the high friction model. In figure $11 \mathrm{~b}$ ) the corresponding comparison is made in the wall normal direction. Here the difference between the high and low friction cases is very small (this small difference matches closely to the results of periodic boundary conditions). We conclude that the presence of the perfectly smooth flat surface enhances the effect of hydrodynamic interactions in the parallel direction.

\section{Conclusion}

We have investigated the accuracy of a Brownian dynamics of reflected random walks as a model to describe the motion of polymer chains in melts near a flat surface. As for the Rouse model there are only two phenomenological parameters: the number of bonds forming a chain, and the bead friction coefficient. We map to this single-chain 'modified-Rouse' model from multi-chain MD models which capture the dominant behaviour of flexible chains. The 
same mapping parameters are used across all bulk and surface observables, in order to test the generality of the modified-Rouse description. Within the MD models, near a flat surface the deviations of chain orientation statistics from reflected random walks are similar to those of chains in bulk from unbounded random walks. In turn, we find that modified-Rouse describes the end-to-end vector correlation function of non-overlapping surface-tethered chains within KG MD as well as the Rouse model does for unconfined free chains. This success follows from the fact that the model accurately describes the correlation functions of the longest Rouse modes, which dominate the end-to-end vector correlation function. The longest Rouse mode correlation function of a surface-tethered chain at low grafting density is well modelled by a reflected Ornstein-Uhlenbeck process, and the ratio of longest relaxation times in wall normal (bounded) and wall parallel (unbounded) directions is $\tau_{\perp} / \tau_{\|}=1 / 2$. We have demonstrated that this good agreement remains when momentum-conservation is approximately restored, by reducing the thermostat friction coefficient by an order of magnitude.

As has been widely observed with the Rouse model, the modified-Rouse model does not describe so well the correlation functions of faster modes. Despite this, far from a surface, the mid-bead mean square displacement of free chains, which depends more strongly on the faster mode dynamics, is reasonably well described by the Rouse model. Using a perfectly smooth flat surface, modified-Rouse simultaneously predicts the mid-bead mean square displacement at $t \approx \tau_{R}$ for both soft MD chains in the immediate surface vicinity and in the channel centre. However the KG MD model predicts a weaker relative drop in bead mobility at the surface compared to the modified-Rouse mapping. These results appear to suggest a weakened topological interaction at the surface compared to in bulk, even before the onset of the entanglement regime. ${ }^{60}$ However, such a clear conclusion cannot be made without more detailed investigations into the effect of the viscoelastic-hydrodynamic coupling on the Soft MD system.

We find that the most important deviation from the simple Rouse picture near to the surface is the neglection of hydrodynamic interactions (HI). However the HI effects are only 
very strong for the zeroth mode dynamics, already described theoretically and investigated with simulation by Farago et al. ${ }^{30}$ For a smooth flat surface we observe an increase in the strength of HI closer to the surface in the parallel direction, apparent through the stronger super-diffusive regime of the centre of mass mean square displacement observable; although in the wall normal direction the strength of HI shows little channel position dependence. Preliminary investigations suggest that the perfectly smooth surface represents the limit of enhanced HI effects at the surface: a roughened surface reduces the super-diffusive centre of mass motion in the surface parallel direction. However, even in the flat wall case investigated here, since the simple modified-Rouse picture does a reasonable job of describing the correlation functions of Rouse modes for $p>0$, the model is still useful for several interesting quantities. This suggests that the simple modified-Rouse description of surface dynamics may be experimentally relevant. The most straightforward test might be to compare the dynamic structure factor of chains in a thin (but weakly confined) film with the prediction of modified-Rouse.

As a single-chain model, the modified-Rouse will not necessarily describe stress relaxation of films, since this generally necessitates a complete description of how contributions to the stress due to orientation cross-correlations between chains at different relative surface positions decay; although for bulk blends Cao et al ${ }^{49}$ have shown that the decay of crosscorrelations between different polymer species may be understood in terms of the decay of auto-correlations. A similar phenomenological attempt at adapting a single chain model to include the cross-correlations between surface and bulk chains was recently made by Abberton et al. ${ }^{61}$ The relevance of the simple modified-Rouse description may be useful to this end.

Additionally, there is a lot of interest in the dynamics of tethered chains at higher grafting density. ${ }^{46,62}$ At higher grafting densities eqns (1), (2), and (3) may give a good description using an appropriate potential. ${ }^{63-66}$ However, it is expected that entanglements between neighbouring grafted chains, and therefore deviations from this simple single chain picture, 
will occur at lower molecular weights in this case. ${ }^{67}$

\section{Acknowledgement}

This work is dedicated to our mentor, colleague and friend, Prof. Alexei E. Likhtman, who contributed greatly to it and continues to inspire future work.

We thank Zuowei Wang for useful discussions and for providing some of the data used in figure 9 c). This work was supported by the Engineering and Physical Sciences Research Council (EPSRC), grant ref: EP/K503125/1. P.I is supported by EU FP7-MC-CIG Grant No. 631233.

\section{Supporting Information}

Random walk boundary value problem solved in the $L \rightarrow \infty$ limit, derivation of eqn (7), derivation of eqn (63), large $\mathrm{N}$ limiting behaviour of $\Phi_{\perp}(t)$ and Rouse mode cross-correlation functions for a surface tethered chain using the modified-Rouse model, and effect of surface nematic interaction.

\section{Random walk boundary value problem}

The propagator $G_{n}\left(y_{1}, y_{0}\right)$ for a discrete random walk in one of three dimensions, beginning at position $y_{0}$, reaching $y_{1}$ in $n$ steps with step-length $l$ satisfies:

$$
G_{n}\left(y_{1}, y_{0}\right)=\frac{G_{n-1}\left(y_{1}-l, y_{0}\right)}{6}+\frac{G_{n-1}\left(y_{1}+l, y_{0}\right)}{6}+\frac{4 G_{n-1}\left(y_{1}, y_{0}\right)}{6}
$$

Using the initial condition $G_{0}\left(y_{1}, y_{0}\right)=\delta\left(y_{1}-y_{0}\right)$, we get $G_{n}\left(y_{1}, y_{0}\right)=\Omega_{n}\left(y_{1}, y_{0}\right) / \tilde{\Omega}_{n}$ where $\Omega_{n}\left(y_{1}, y_{0}\right)$ is the number of walks (partition function) between $y_{0}$ and $y_{1}$ with $n$ steps and $\tilde{\Omega}_{n}$ is the total number of walks with $n$ steps and fixed start position in a system whereby at each step there are six distinct moves with a $100 \%$ survival probability. In the limit $n \rightarrow \infty$, 
$l \rightarrow 0$, from eqn (33) the propagator diffusion equation in the wall normal direction is found:

$$
\frac{\partial G_{n}\left(y_{1}, y_{0}\right)}{\partial n}=\frac{l^{2}}{6} \frac{\partial^{2} G_{n}\left(y_{1}, y_{0}\right)}{\partial y_{1}^{2}}
$$

We seek the solution of eqn (34) which satisfies reflective boundary conditions at $y_{1}=0, L$ :

$$
\left.\frac{\partial G_{n}\left(y_{1}, y_{0}\right)}{\partial y_{1}}\right|_{y_{1}=0, L}=0
$$

in the limit $L \rightarrow \infty$. Using this boundary condition, the total number of walks inside the channel is always conserved and equal to $\tilde{\Omega}_{n}$, so that the propagator must be a probability under the initial condition that the walk began at $y_{0}$.

The general solution of eqn (34) subject to reflective boundaries at 0 and $L$ is well known, consisting of $2 m$ solutions with eigenvalues $\lambda_{m}$ :

$$
\sum_{m}\left(B_{m} \cos \left(\sqrt{\lambda_{m}} y_{1}\right)+D_{m} \sin \left(\sqrt{\lambda_{m}} y_{1}\right)\right) \exp \left(-\sigma_{1}^{2} \lambda_{m} / 2\right)
$$

where $\sigma_{1}^{2}=b^{2} / 3=l^{2} n / 3 . \quad b$ is the characteristic length scale of the random walk. Applying the boundary conditions enforce $D_{m}=0$, and $\lambda_{m}=\left(\frac{\pi m}{L}\right)^{2}: \quad m=0,1,2,3, \ldots, \infty$. Now we apply the initial condition: $G_{0}\left(y_{1}, y_{0}\right)=\delta\left(y_{1}-y_{0}\right)$ :

$$
\delta\left(y_{1}-y_{0}\right)=\sum_{m} B_{m} \cos \left(\sqrt{\lambda_{m}} y_{1}\right)
$$

Then the $B_{m}$ are found as:

$$
B_{m}=\frac{2}{L} \int_{0}^{L} \cos \left(\sqrt{\lambda_{m}} y_{1}\right) \delta\left(y_{1}-y_{0}\right) d y_{1}=\frac{2}{L} \cos \left(\sqrt{\lambda_{m}} y_{0}\right)
$$

The complete solution is then:

$$
\sum_{m=0}^{\infty} \frac{2}{L} \cos \left(\sqrt{\lambda_{m}} y_{0}\right) \cos \left(\sqrt{\lambda_{m}} y_{1}\right) \exp \left(-\sigma_{1}^{2} \lambda_{m} / 2\right)=\sum_{m=0}^{\infty} f(m)
$$


From eqn (39), $f^{\prime}(m) \rightarrow 0$ for $L \rightarrow \infty$ over the whole range of $m$. Resultantly, for $L \rightarrow \infty$ $f(m)$ is a constant over any finite interval, and the sum, eqn (39), may be equated with the following integral:

$$
\begin{aligned}
& \lim _{L \rightarrow \infty} \frac{2}{L} \int_{0}^{\infty} d m \cos \left(\sqrt{\lambda_{m}} y_{0}\right) \cos \left(\sqrt{\lambda_{m}} y_{1}\right) \exp \left(-\sigma_{1}^{2} \lambda_{m} / 2\right)= \\
& \lim _{L \rightarrow \infty} \frac{1}{L} \int_{0}^{\infty} d m\left(\cos \left(\sqrt{\lambda_{m}}\left(y_{1}+y_{0}\right)\right)+\cos \left(\sqrt{\lambda_{m}}\left(y_{1}-y_{0}\right)\right)\right) \exp \left(-\sigma_{1}^{2} \lambda_{m} / 2\right)
\end{aligned}
$$

Rewriting this integral in the following form:

$$
\int_{0}^{\infty} d U \cos (a U) \exp \left(-U^{2}\right)=\frac{\sqrt{\pi}}{2} \exp \left(-\frac{a^{2}}{4}\right)
$$

and evaluating, leads to the solution for the propagator (conditional probability):

$$
\lim _{L \rightarrow \infty} P\left(y_{1} \mid y_{0}, L\right)=\frac{1}{\sqrt{2 \pi \sigma_{1}^{2}}} \exp \left(-\frac{\left(y_{1}-y_{0}\right)^{2}}{2 \sigma_{1}^{2}}\right)+\frac{1}{\sqrt{2 \pi \sigma_{1}^{2}}} \exp \left(-\frac{\left(y_{1}+y_{0}\right)^{2}}{2 \sigma_{1}^{2}}\right)
$$

Translating the coordinates such that the surface position is translated to $y_{w}$, we find a general expression in terms of unbounded random walk probability weights:

$$
\begin{aligned}
& P\left(y_{1} \mid y_{0}, y_{w}\right)=P\left(y_{1} \mid y_{0}\right)+P\left(\overline{y_{1}} \mid y_{0}\right)= \\
& \frac{1}{\sqrt{2 \pi \sigma_{1}^{2}}} \exp \left(-\frac{\left(y_{1}-y_{0}\right)^{2}}{2 \sigma_{1}^{2}}\right) \\
& +\frac{1}{\sqrt{2 \pi \sigma_{1}^{2}}} \exp \left(-\frac{\left(-\tilde{y}_{1}-\tilde{y}_{0}\right)^{2}}{2 \sigma_{1}^{2}}\right)
\end{aligned}
$$

where $\tilde{y}_{i}=y_{i}-y_{w}$.

\section{Free energy of a reflected random walk}

We now assume that near a flat surface the probability distribution for positions of the start and end beads of polymer chain sub-segments in the canonical ensemble at arbitrary 
temperature $T$ coincides with a reflected random walk. We give temperature in units of $k_{B}$. Then a conformational free energy may be defined for the polymer chain. The partition function, $\Omega\left(\mathbf{R}_{i}, \mathbf{R}_{i-1}, y_{w}\right)$, for a three dimensional random walk from $\mathbf{R}_{i-1}$ to $\mathbf{R}_{i}$ in the presence of a reflective surface at position $y_{w}$, can be written in terms of a sum of the probabilities of unbounded random walks of bond vector $\mathbf{Q}_{i}=\mathbf{R}_{i}-\mathbf{R}_{i-1}$ and bond vector from $\mathbf{R}_{i-1}$ to the reflection of $\mathbf{R}_{i}$ in the $y=y_{w}$ plane, $\overline{\mathbf{Q}}_{i}$ :

$$
\Omega\left(\mathbf{R}_{i}, \mathbf{R}_{i-1}, y_{w}\right)=\left(P\left(\mathbf{Q}_{i}\right)+P\left(\overline{\mathbf{Q}}_{i}\right)\right) \tilde{\Omega}
$$

where $\tilde{\Omega}$ is the total number of walks with fixed start. Therefore the thermodynamic potential (conformational free energy) may be defined up to a constant as:

$$
U\left(\mathbf{Q}_{i}, \overline{\mathbf{Q}}_{i}\right)=-T \ln \left(P\left(\mathbf{Q}_{i}\right)+P\left(\overline{\mathbf{Q}}_{i}\right)\right)
$$

Identifying the symmetric (bonded) part of the potential as $-T \ln \left(P\left(\mathbf{Q}_{i}\right)\right)=\frac{k}{2} \mathbf{Q}_{i} \cdot \mathbf{Q}_{i}$, where $k=T / \sigma_{1}^{2}$, eqn (45) can be written as a sum of a bonded and non-bonded potential:

$$
U\left(\mathbf{Q}_{i}, \overline{\mathbf{Q}}_{i}\right)=-T\left(\ln \left(P\left(\mathbf{Q}_{i}\right)\right)+\ln \left(1+\frac{P\left(\overline{\mathbf{Q}}_{i}\right)}{P\left(\mathbf{Q}_{i}\right)}\right)\right)
$$

The bond vectors may be resolved into components parallel to and normal to the surface: $\mathbf{Q}_{i}=\mathbf{Q}_{i, \|}+\left(y_{i}-y_{i-1}\right) \hat{\mathbf{e}}_{\perp} ; \quad \overline{\mathbf{Q}}_{i}=\mathbf{Q}_{i, \|}+\left(-\tilde{y}_{i}-\tilde{y}_{i-1}\right) \hat{\mathbf{e}}_{\perp}$. Since $\mathbf{Q}_{i}$ and $\overline{\mathbf{Q}}_{i}$ only differ in the wall normal direction, the quotient in eqn (46) is:

$$
\begin{aligned}
\frac{P\left(\overline{\mathbf{Q}}_{i}\right)}{P\left(\mathbf{Q}_{i}\right)}= & \exp \left(-\frac{\left(\left(-\tilde{y}_{i}-\tilde{y}_{i-1}\right)^{2}-\left(y_{i}-y_{i-1}\right)^{2}\right)}{2 \sigma_{1}^{2}}\right)= \\
& \exp \left(-\frac{2 \tilde{y}_{i} \tilde{y}_{i-1}}{\sigma_{1}^{2}}\right)
\end{aligned}
$$


Therefore the thermodynamic potential up to a constant is:

$$
U\left(\mathbf{Q}_{i}, \overline{\mathbf{Q}}_{i}\right)=\frac{k}{2} \mathbf{Q}_{i} \cdot \mathbf{Q}_{i}+A\left(y_{i}, y_{i-1}, y_{w}\right)
$$

where the wall contribution is:

$$
A\left(y_{i}, y_{i-1}, y_{w}\right)=-T \ln \left(1+\exp \left(-\frac{2 \tilde{y}_{i} \tilde{y}_{i-1}}{\sigma_{1}^{2}}\right)\right)
$$

The additional thermodynamic force on particle $i$ in the wall normal direction can now be calculated:

$$
-\frac{\partial A\left(y_{i}, y_{i-1}, y_{w}\right)}{\partial \tilde{y}_{i}}=-\frac{\exp \left(-\frac{2 \tilde{y}_{i} \tilde{y}_{i-1}}{\sigma_{1}^{2}}\right)}{1+\exp \left(-\frac{2 \tilde{y}_{i} \tilde{y}_{i-1}}{\sigma_{1}^{2}}\right)} 2 k \tilde{y}_{i-1}
$$

In the case of absorbing boundary condition, the partition function for a chain beginning at $\mathbf{R}_{i-1}$ and ending at $\mathbf{R}_{i}$ has to be zero when either bead is at the surface. This is satisfied with the propagator $G_{n}\left(y_{1}, y_{0}\right)=P\left(\mathbf{Q}_{i}\right)-P\left(\overline{\mathbf{Q}}_{i}\right):{ }^{39}$

$$
\Omega\left(\mathbf{R}_{i}, \mathbf{R}_{i-1}, y_{w}\right)=\left(P\left(\mathbf{Q}_{i}\right)-P\left(\overline{\mathbf{Q}}_{i}\right)\right) \tilde{\Omega}
$$

Resultantly, the corresponding wall potential has a singularity at $\tilde{y}_{i}, \tilde{y}_{i-1}=0$ :

$$
B\left(y_{i}, y_{i-1}, y_{w}\right)=-T \ln \left(1-\exp \left(-\frac{2 \tilde{y}_{i} \tilde{y}_{i-1}}{\sigma_{1}^{2}}\right)\right)
$$

\section{End-to-end vector correlation function of a dumbbell teth- ered at a reflective surface}

The probability distribution of the end-to-end vector of a reflected walk in the surface normal direction, eqn (43), becomes a reflected-Gaussian for $y_{0}=y_{w}$. Resultantly, when modelling 
the wall grafted chain using a single dumbbell, the potential reduces to the simple harmonic form, $U\left(y_{1}\right)=k / 2\left(y_{1}-y_{w}\right)^{2}$, such that the mean square end-to-end distance of the whole chain (dumbbell) is related to the spring constant via the equipartition theorem as: $\left\langle\left(y_{1}-y_{w}\right)^{2}\right\rangle=T / k$. Setting $y_{w}=0$, the first two moments of the half Gaussian end bead distribution are:

$$
\begin{aligned}
& \left\langle y_{1}\right\rangle=\sqrt{2 \sigma_{1}^{2} / \pi} \\
& \left\langle y_{1}^{2}\right\rangle=\sigma_{1}^{2}
\end{aligned}
$$

Knowing these moments, we only need to calculate the equilibrium correlation function of the end bead vector:

$$
\left\langle y_{1}(t) y_{1}(0)\right\rangle=\int_{0}^{\infty} d y_{1}^{\prime} y_{1}^{\prime} \Psi_{\mathrm{eq}}\left(y_{1}^{\prime}\right) \int_{0}^{\infty} d y_{1} y_{1} G_{t}\left(y_{1}, y_{1}^{\prime}\right)
$$

in order to solve the dumbbell end-to-end vector correlation function $\Phi_{\mathrm{db}, \perp}(t)$ :

$$
\Phi_{\mathrm{db}, \perp}(t)=\frac{\left\langle y_{1}(t) y_{1}(0)\right\rangle-\left\langle y_{1}\right\rangle^{2}}{\left\langle y_{1}^{2}\right\rangle-\left\langle y_{1}\right\rangle^{2}}
$$

$\Psi_{\text {eq }}\left(y_{1}^{\prime}\right)$ is the equilibrium distribution function of the end position and $G_{t}\left(y_{1}, y_{1}^{\prime}\right)$ is the propagator of an end bead with position $y_{1}^{\prime}$ at time 0 and position $y_{1}$ at time $t$, corresponding to the system described by the Langevin dynamics:

$$
\frac{d y_{1}}{d t}=\frac{1}{\xi}\left(-\frac{\partial U}{\partial y_{1}}+f^{r}(t)\right)
$$

supplemented with a reflective boundary; where $\xi$ is the free bead friction and $f^{r}(t)$ is a white-noise random variable with zero mean and variance $2 \xi T$. The propagator satisfies the Smoluchowski equation:

$$
\frac{\partial G_{t}\left(y_{1}, y_{1}^{\prime}\right)}{\partial t}=\frac{1}{\xi} \frac{\partial}{\partial y_{1}}\left(T \frac{\partial G_{t}\left(y_{1}, y_{1}^{\prime}\right)}{\partial y_{1}}+\frac{\partial U}{\partial y_{1}} G_{t}\left(y_{1}, y_{1}^{\prime}\right)\right)
$$


Using the potential $U\left(y_{1}\right)=(k / 2) y_{1}^{2}$, eqn (56) becomes the Ornstein-Uhlenbeck process. Then the solution of eqn (57) over the unbounded domain, $(-\infty, \infty)$, is ${ }^{27}$

$$
\tilde{G}_{t}\left(y_{1}, y_{1}^{\prime}\right)=\frac{1}{\sqrt{2 \pi \sigma^{2}(t)}} \exp \left(-\frac{\left(y_{1}-y_{1}^{\prime} \exp \left(-t / \tau_{\mathrm{db}, \|}\right)\right)^{2}}{2 \sigma^{2}(t)}\right)
$$

where $\sigma(t)=\sigma_{1} \sqrt{1-\exp \left(-2 t / \tau_{\mathrm{db}, \|}\right)}$ and $\tau_{\mathrm{db}, \|}=\xi / k$ is the relaxation time of the unbounded system. We seek the solution of the propagator under a reflective boundary condition:

$$
\left.\frac{\partial G_{t}\left(y_{1}, y_{1}^{\prime}\right)}{\partial y_{1}}\right|_{y_{1}=0}=0
$$

Due to the symmetry of the harmonic potential, we can construct the solution, $G_{t}\left(y_{1}, y_{1}^{\prime}\right)$, satisfying eqn (57) and the reflective boundary condition, eqn (59), from the solution $\tilde{G}_{t}\left(y_{1}, y_{1}^{\prime}\right)$, as:

$$
G_{t}\left(y_{1}, y_{1}^{\prime}\right)=\tilde{G}_{t}\left(y_{1}, y_{1}^{\prime}\right)+\tilde{G}_{t}\left(-y_{1}, y_{1}^{\prime}\right)
$$

Using this propagator, an arbitrary probability distribution becomes the equilibrium halfGaussian distribution for $t \rightarrow \infty$ :

$$
\begin{aligned}
\Psi_{\text {eq }}\left(y_{1}\right) & =\lim _{t \rightarrow \infty} \int_{0}^{\infty} d y_{1}^{\prime}\left(\tilde{G}_{t}\left(y_{1}, y_{1}^{\prime}\right)+\tilde{G}_{t}\left(-y_{1}, y_{1}^{\prime}\right)\right) \Psi_{0}\left(y_{1}^{\prime}\right) \\
& =2\left(\frac{1}{\sqrt{2 \pi \sigma_{1}^{2}}} \exp \left(-\frac{y_{1}^{2}}{2 \sigma_{1}^{2}}\right)\right)
\end{aligned}
$$

$\Psi_{0}\left(y_{1}^{\prime}\right)$ is the probability for the particle to be at position $y_{1}^{\prime}$ at an initial time when the distribution may not have reached equilibrium. Now eqn (54) may be written as:

$$
\left\langle y_{1}(t) y_{1}(0)\right\rangle=\int_{0}^{\infty} d y_{1}^{\prime} y_{1}^{\prime} \Psi_{\mathrm{eq}}\left(y_{1}^{\prime}\right) \int_{0}^{\infty} d y_{1} y_{1}\left(\tilde{G}_{t}\left(y_{1}, y_{1}^{\prime}\right)+\tilde{G}_{t}\left(-y_{1}, y_{1}^{\prime}\right)\right)
$$


Carrying out the integrals in eqn (62) leads after some calculation to $\Phi_{\mathrm{db}, \perp}(t)$ via eqn (55):

$$
\Phi_{\mathrm{db}, \perp}(t)=\frac{1}{\pi / 2-1}\left(\arctan \left(\mathrm{e}^{-\frac{t}{\tau_{\mathrm{db},}, \|}} \frac{1}{\sqrt{1-\mathrm{e}^{-\frac{2 t}{\tau_{\mathrm{db},}, \|}}}}\right) \mathrm{e}^{-\frac{t}{\tau_{\mathrm{db}, \|}}}+\sqrt{1-\mathrm{e}^{-\frac{2 t}{\tau_{\mathrm{db}, \|}}}}-1\right)
$$

\section{Modified Rouse model - large N limiting behaviour of $\Phi_{\perp}(t)$}

As the number of bonds in a chain diverges, $N \rightarrow \infty$, the functional form of $\Phi_{\perp}(t)$ for a surface tethered-chain converges at a similar rate as for the surface parallel case, which is described by standard Rouse theory. In figure 13, $\Phi_{\perp}(t)$ and $\Phi_{\|}(t)$ are compared for $N=1,2,4,8,16,32$. For each plot the time axis is normalized by the corresponding longest system relaxation time $\tau_{1, \alpha}$, which in the surface parallel direction is: ${ }^{47}$

$$
\tau_{1, \|}=\frac{\xi b^{2}}{12 T \sin ^{2}\left(\frac{\pi(1 / 2)}{2(N+1 / 2)}\right)}
$$

In the surface normal direction $\tau_{1, \perp}$ is found by assuming that the relationship:

$$
\frac{\tau_{1, \perp}}{\tau_{1, \|}}=\frac{\tau_{\mathrm{db}, \perp}}{\tau_{\mathrm{db}, \|}}=\frac{1}{2}
$$

is valid for all $N$. From figure 13 it is apparent that the changes in the form of $\Phi_{\perp}(t)$ as $N$ increases from $N=1$ are greater than in the unbounded direction. This can be largely attributed to a decrease of the relative weight of the longest Rouse mode amplitude which does not dominate $\Phi_{\perp}(t)$ as much as it does $\Phi_{\|}(t)$. In general, the amplitudes of the modes strongly differ from the wall parallel direction. The first five Rouse mode amplitudes of reflected random walks beginning at the surface are plotted and compared to KG MD in section 3 of the main letter. 


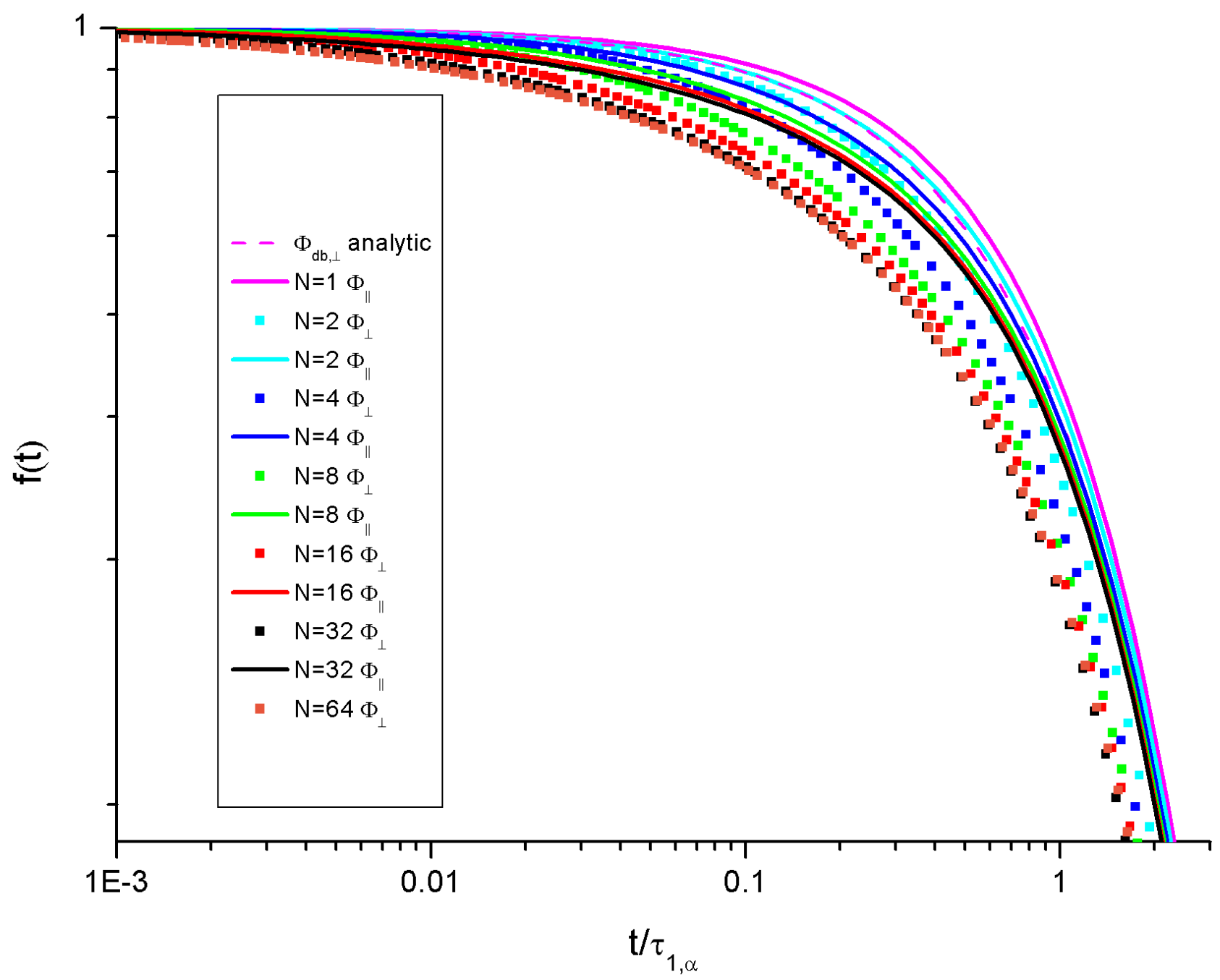

Figure 13: Comparison of wall normal (symbols) and parallel (lines) components of $\Phi_{\alpha}(t)$ for a chain modelled using different numbers of bonds. Both directions give converged behaviour beyond $N \approx 32$. Time axes are normalized by the longest relaxation time in the corresponding direction. 


\section{Rouse mode cross-correlation functions for a surface tethered chain}

Since in a confined system the Rouse coordinates are no longer normal modes, their crosscorrelations are no longer zero. Here we simply present the qualitative behaviour of crosscorrelations within the modified-Rouse model of a surface tethered chain (this qualitative behaviour is shared by KG MD), and show that the relative importance of cross-correlations is small compared to auto-correlations.

A general characteristic of the Rouse coordinates of a reflected random walk beginning at the surface is that the covariances, $\left\langle X_{p, \perp} X_{q, \perp}\right\rangle-\left\langle X_{p, \perp}\right\rangle\left\langle X_{q, \perp}\right\rangle$, are negative. This is an observation we make for all cases considered and is not generally proven. Since the mode covariances coincide with the amplitudes of the mode cross-correlation functions:

$$
C_{p q, \perp}(t)=\left\langle\left(X_{p, \perp}(t)-\left\langle X_{p, \perp}\right\rangle\right)\left(X_{q, \perp}(0)-\left\langle X_{q, \perp}\right\rangle\right)\right\rangle
$$

these functions are increasing. Figure 14 shows that the magnitude of the sum over all first mode covariances quickly converges to a effectively constant value as $N$ is increased. This value is more than seven times smaller than the variance of the first mode. The relative importance of cross-correlations is a decreasing function of $p$.

\section{Effect of surface nematic interaction}

In this section we investigate the weak additional swelling in the chain dimension apparent in figure 6 of the main letter, where it was found that the mean square end-to-end distance of complete chains comprising $n=64$ bonds is around $10 \%$ larger for KG MD chains beginning with one end at the surface, compared to in the channel centre. We partially attribute this swelling to the influence of the surface on short range nematic ordering. We consider a nematic order parameter of chain sub-segments comprising $m=4$ bonds. The nematic 


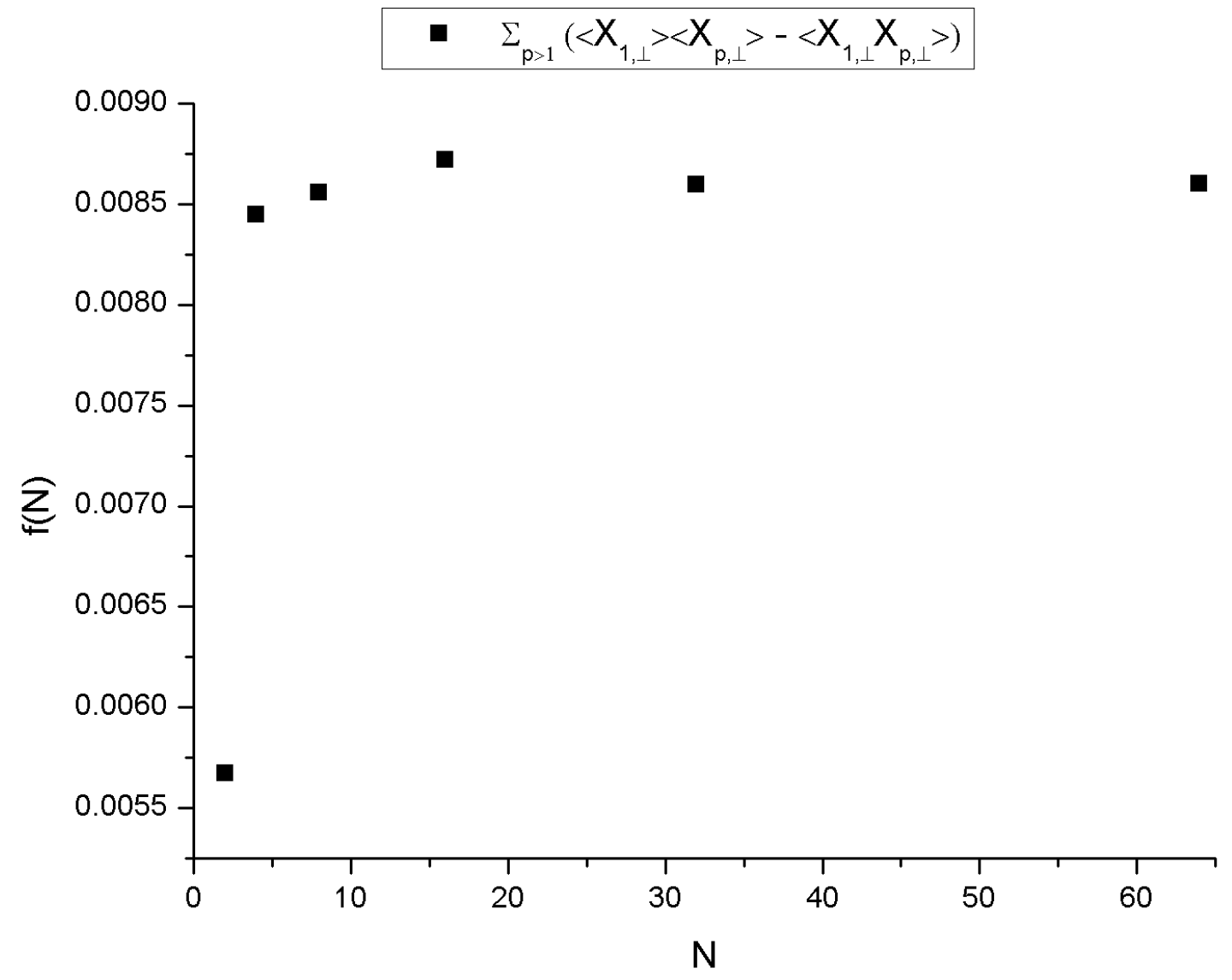

Figure 14: Negative of the sum over all first mode covariances as a function of the number of bonds in a chain, normalized by the bulk chain mean square end-to-end distance in one direction. 
order parameter is:

$$
S_{4, \|}(y)=\left\langle P_{2}\left(\hat{k}_{4, \|}(y)\right)\right\rangle
$$

where $\hat{k}_{4, \|}$ is a parallel component of the coarse-grained bond unit vector corresponding to a chain segment consisting of 4 bonds. $P_{2}(x)=\frac{3 x^{2}-1}{2}$ is the second Legendre polynomial, and the averages are performed over bins centred at position $y=\Delta y(i+1 / 2)$ for $i=0,1,2,3, \ldots$; where $\Delta y$ is the bin width. Each sub-segment is binned depending on the distance from the wall of the sub-segment end-bead position.

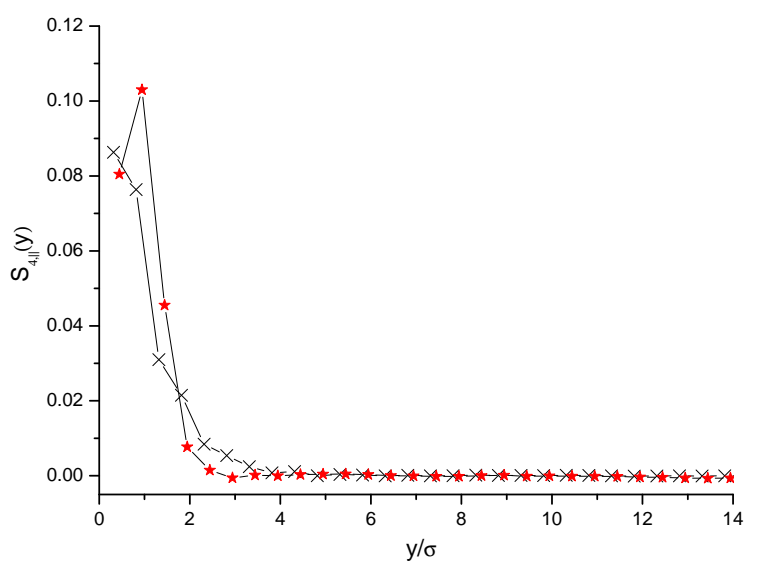

Figure 15: Nematic order parameter for chain sub-segments comprising $m=4$ bonds. Binned by sub-segment end position. Red stars: KG MD; black crosses: soft MD.

It is necessary to use coarse-grained segments since for $m<4$ the wall ordering obscures the trend near to the surface. In figure 15 we plot the nematic order parameter, $S_{4, \|}(y)$, as a function of the centre bin position. The decay in $S_{4, \|}(y)$ occurs over a distance $\approx 2 \sigma$ from the surface, which is similar to an approximate blob length for the Kremer-Grest chains. For semi-flexible polymers the nematic ordering persists over a longer interval. ${ }^{68}$ Since the density of the centre of mass within a blob length from the surface for such short chains is non-negligible, it is likely that there are also contributions to the chain swelling due to long range correlations ${ }^{34}$ for a non-negligible proportion of the chains near the surface. The short range nematic order parameter corresponding to soft MD chains, also plotted in figure 15, 
is no greater than KG MD at the surface. The higher density of ends immediately at the surface $^{58}$ is also expected to influence the surface normal square internal distance profiles in some way.

\section{References}

(1) Varnik, F.; Binder, K. Multiscale modeling of polymers at interfaces. Int. Journ. Mat. Sci. 2009, 100, 1494-1502.

(2) Weir, M. P.; Johnson, D. W.; Boothroyd, S. C.; Savage, R. C.; Thompson, R. L.; King, S. M.; Rogers, S. E.; Coleman, K. S.; Clarke, N. Distortion of Chain Conformation and Reduced Entanglement in Polymer-Graphene Oxide Nanocomposites. ACS. Macro. Lett. 2016, 5, 430-434.

(3) Vladkov, M. Modeling interfacial effects on transport properties: mechanical properties of polymers, thermal properties of nanofluids. Ph.D. thesis, 2007.

(4) Black, W. B.; Graham, M. D. Wall-slip and polymer melt flow instability. Phys. Rev. Lett. 1996, 77\%, 956-959.

(5) Tchesnokov, M. A.; Molenaar, J.; Slot, J. J. M.; Stepanyan, R. A molecular model for cohesive slip at polymer melt/ solid interfaces. J. Chem. Phys. 2005, 122, 214711.

(6) Hoy, R. S.; Grest, G. S. Entanglements of an End-Grafted Polymer Brush in a Polymeric Matrix. Macromolecules 2007, 40, 8389-8395.

(7) Sethuraman, V.; Kipp, D.; Ganesan, V. Entanglements in Lamellar Phases of Diblock Copolymers. Macromolecules 2015, 48, 6321-6328.

(8) Sussman, D. M.; Tung, W.-S.; Winey, K. I.; Schweizer, K. S.; Riggleman, R. A. Entanglement Reduction and Anisotropic Chain and Primitive Path Conformations in Polymer Melts under Thin Film and Cylindrical Confinement. Macromolecules 2014, 47, 6462-6472. 
(9) Rouse Jr, P. E. A theory of the linear viscoelastic properties of dilute solutions of coiling polymers. J. Chem. Phys. 1953, 21, 1272.

(10) Solar, M.; Yelash, L.; Virnau, B.; Binder, K.; Paul, W. Polymer Dynamics in a PolymerSolid Interphase: Molecular Dynamics Simulations of 1,4-Polybutadiene At a Graphite Surface. Soft Materials 2014, 12, S80-S89.

(11) Kremer, F. Dynamics in Geometrical Confinement; Advances in Dielectrics; Springer, 2014.

(12) Mischler, C.; Baschnagel, J.; Binder, K. Polymer Films in the Normal-Liquid and Supercooled State: A Review of Recent Monte Carlo Simulation Results. Adv. Coll. Int. Sci. 2001, 94, 197-227.

(13) Yelash, L.; Virnau, P.; Binder, K.; Paul, W. A slow process in confined polymer melts: layer exchange dynamics at a polymer solid interface. Phys. Rev. E. 2010, 82, 050801.

(14) Vladkov, M.; Barrat, J.-L. Local Dynamics and Primitive Path Analysis for a Model Polymer Melt near a Surface. Macromolecules 2007, 40, 3797-3804.

(15) Smith, K. A.; Vladkov, M.; Barrat, J.-L. Polymer Melt near a Solid Surface. Macromolecules 2005, 38, 571-580.

(16) Kremer, K.; Grest, G. S. Dynamics of entangled linear polymer melts: A moleculardynamics simulation. J. Chem. Phys. 1990, 92, 5057.

(17) Thompson, P. A.; Robbins, M. O. Shear flow near solids: Epitaxial ordering and flow boundary conditions. Phys. Rev. A. 1990, 41, 6830-6837.

(18) Sarabadani, J.; Milchev, A.; Vilgis, T. Molecular Dynamic Study of the Structure and Dynamics of Polymer Melt at Solid Surfaces. J. Chem. Phys. 2014, 141, 044907.

(19) Ferry, J. D. Viscoelastic properties of polymers; New York : Wiley, 1980. 
(20) McLeish, T. C. B. Tube theory of entangled polymer dynamics. Adv. Phys. 2002, 51, $1379-1527$.

(21) Paul, W.; Smith, G. D. Structure and dynamics of amorphous polymers: computer simulations compared to experiment and theory. Reports on Progress in Physics 2004, 67, 1117-1185.

(22) Paul, W.; Smith, G. D.; Yoon, D. Y.; Farago, B.; Rathgeber, S.; Zirkel, A.; Willner, L.; Richter, D. Chain motion in an unentangled polyethylene melt: A critical test of the rouse model by molecular dynamics simulations and neutron spin echo spectroscopy. Phys. Rev. Lett. 1998, 80, 2346-2349.

(23) Kalathi, J. T.; Kumar, S. K.; Rubinstein, M.; Grest, G. S. Rouse mode analysis of chain relaxation in homopolymer melts. Macromolecules 2014, 47, 6925-6931.

(24) Flory, P. J. Principles of Polymer Chemistry; Cornell University Press: Ithaca, NY, 1953.

(25) Wittmer, J. P.; Meyer, H.; Johner, A.; Obukhov, S.; Mattioni, L.; Müller, M.; Semenov, A. N. Long Range Bond-Bond Correlations in Dense Polymer Solutions. Phys. Rev. Lett. 2004, 93, 147801.

(26) Wittmer, J. P.; Beckrich, P.; Johner, J.; Semenov, A. N.; Obukhov, S. P.; Meyer, H.; Baschnagel, J. Why polymer chains in a melt are not random walks. EPL 2007, 77, 56003.

(27) Doi, M.; Edwards, S. F. The Theory of Polymer Dynamics; Clarendon Press Oxford, 1986.

(28) Fatkullin, N. F.; Shakirov, T. M.; Balakirev, N. A. Why does the rouse model fairly describe the dynamic characteristics of polymer melts at molecular masses below critical mass? POLYMER SCIENCE SERIES A 2010, 52, 72-81. 
(29) Farago, J.; Meyer, H.; Baschnagel, J.; N Semenov, A. Hydrodynamic and viscoelastic effects in polymer diffusion. Journal of Physics: Condensed Matter 2012, 24, 284105284115.

(30) Farago, J.; Meyer, H.; Baschnagel., J.; Semenov, A. N. Mode-coupling approach to polymer diffusion in an unentangled melt. II. The effect of viscoelastic hydrodynamic interactions. Phys. Rev. E. 2012, 85, 051807.

(31) Likhtman, A. E. Whither tube theory. J. Non-Newt. Fluid. Mech. 2009, 157, 158-161.

(32) Farago, J.; Semenov, A. N.; Meyer, H.; Wittmer, J. P.; Johner, A.; Baschnagel, J. Mode-coupling approach to polymer diffusion in an unentangled melt. I. The effect of density fluctuations. Phys. Rev. E. 2012, 85, 051806.

(33) Semenov, A. N.; Johner, A. Theoretical notes on dense polymers in two dimensions. Eur. Phys. J. E 2003, 12, 469-480.

(34) Cavallo, A.; Müller, M.; Wittmer, J. P.; Johner, A.; Binder, K. Single chain structure in thin polymer films: corrections to Flory's and Silberberg's hypotheses. Journal of Physics: Condensed Matter 2005, 17, S1697-S1709.

(35) Lee, N.-K.; Farago, J.; Meyer, H.; Wittmer, J. P.; Baschnagel, J.; Obukhov, S. P. Non-ideality of polymer melts confined to nanotubes. EPL 2011, 93, 48002.

(36) Likhtman, A. E. In Polymer Science: A Comprehensive Reference; Matyjaszewski, K., Muller, M., Eds.; Elsevier B.V, 2012; Vol. 10; p 133.

(37) Öttinger, H. C., Stochastic processes in polymeric fluids: tools and examples for developing simulation algorithms; Berlin ; Springer, 1996.

(38) Silberberg, A. Distribution of Conformations and Chain Ends near the Surface of a Melt of Linear Flexible Macromolecules. Journ. Coll. Int. Sci 1981, 90, 86-91. 
(39) Woo, N. J.; Shaqfeh, E. S. G.; Khomani, B. Effect of confinement on dynamics and rheology of dilute DNA solutions. I. Entropic spring force under confinement and a numerical algorithm. J. Rheol. 2004, 48, 281-298.

(40) Peters, E. A. J. F.; Barenbrug, T. M. A. O. M. Efficient Brownian dynamics simulation of particles near walls. I. Reflecting and absorbing walls. Phys. Rev. E 2002, 66, 056701.

(41) Noid, W. G.; Ayton, G. S.; Izvekov, S.; Voth, G. A. In Coarse-Graining of Condensed Phase and Biomolecular Systems; Voth, G. A., Ed.; CRC Press, 2009; p 21.

(42) Ilg, P.; Mavrantzas, V.; Öttinger, H. C. In Modeling and Simulations in Polymers; Purushottam, D. G., Leonov, A. I., Eds.; Wiley, 2009; Vol. 7.

(43) Akkermans, R. L. C.; Briels, W. J. Coarse-grained dynamics of one chain in a polymer melt. J. Chem. Phys. 2000, 113, 6409-6422.

(44) Ilg, P.; Ottinger, H. C.; Kroger, M. Systematic time-scale-bridging molecular dynamics applied to flowing polymer melts. Phys. Rev. E. 2009, 79, 011802.

(45) Wang, S.-Q.; Ravindranath, S.; Boukany, P. E. Homogeneous shear, wall slip, and shear banding of entangled polymeric liquids in simple-shear rheometry: A roadmap of nonlinear rheology. Macromolecules 2011, 44, 183-190.

(46) Singh, M. K.; Ilg, P.; Espinosa-Marzal, R. M.; Kroger, M.; Spencer, N. D. Polymer Brushes under Shear: Molecular Dynamics Simulations Compared to Experiments. Langmuir 2015, 31, 4798-4805.

(47) Likhtman, A. E. Short course: Entangled Polymer Dynamics. http://www . personal.reading.ac.uk/ sms06al2/short\_course.

(48) http://www.reptate.com/.

(49) Cao, J.; Likhtman, A. E. Time-dependent orientation coupling in equilibrium polymer melts. Phys. Rev. Lett. 2010, 104, 207801. 
(50) Cao, J.; Likhtman, A. E. Simulating Startup Shear of Entangled Polymer Melts. ACS. Macro. Lett. 2015, 4, 1376-1381.

(51) Likhtman, A. E.; Sukumaran, S. K.; Ramirez, J. Linear Viscoelasticity from Molecular Dynamics Simulation of Entangled Polymers. Macromolecules 2007, 40, 6748-6757.

(52) Hou, J. X.; Svaneborg, C.; Everaers, R.; Grest, G. S. Stress Relaxation in Entangled Polymer Melts. Phys. Rev. Lett. 2010, 105, 068301.

(53) Auhl, R.; Everaers, R.; Grest, G. S.; Kremer, K.; Plimpton, S. J. Equilibration of Long Chain Polymer Melts in Computer Simulations. J. Chem. Phys. 2003, 119, 12718.

(54) Allen, M. P.; Tildesley, D. J. Computer Simulation of Liquids; Oxford Science Publications, 1987.

(55) Ramírez, J.; Sukumaran, S. K.; Vorselaars, B.; Likhtman, A. E. Efficient on the fly calculation of time correlation functions in computer simulations. J. Chem. Phys. 2010, 133, 154103.

(56) Skvortsov, A. M.; Leermakers, F. A. M.; Fleer, G. J. Equivalence of chain conformations in the surface region of a polymer melt and a single Gaussian chain under critical conditions. J. Chem. Phys. 2014, 139, 054907.

(57) Eisenriegler, E.; Kremer, K.; Binder, K. Adsorption of polymer chains at surfaces: Scaling and Monte Carlo analyses. J. Chem. Phys. 1982, 77, 6296-6320.

(58) Matsen, M. W.; Mahmoudi, P. Segregation of chain ends to the surface of a polymer melt. The European Physical Journal E 2014, 37, 78.

(59) McLeish, T. In Soft and Fragile Matter; Cates, M. E., Evans, M. R., Eds.; Scottish Graduate Textbook Series; IOP, 2000; p 79.

(60) Brown, H. R.; Russell, T. Entanglements at Polymer Surfaces and Interfaces. Macromolecules 1996, 29, 798-800. 
(61) Abberton, B. C.; Liu, W. K.; Keten, S. Anisotropy of Shear Relaxation in Confined Thin Films of Unentangled Polymer Melts. Macromolecules 2015, 48, 7631-7639.

(62) Reith, D.; Milchev, A.; Vernau, P.; Binder, K. Computer Simulation Studies of Chain Dynamics in Polymer Brushes. Macromolecules 2014, 45, 4381-4393.

(63) de Gennes, P. G. Conformations of Polymers Attached to an Interface. Macromolecules 1980, 13, 1069-1075.

(64) Milner, S. T. Polymer Brushes. Science 1991, 251, 905-914.

(65) Likhtman, A. E.; Semenov, A. N. An advance in the theory of strongly segregated polymers. Europhysics Letters 2000, 51, 307-313.

(66) Matsen, M. W. Investigating the dominant corrections to the strong-stretching theory for dry polymeric brushes. J. Chem. Phys. 2004, 121, 1938-1948.

(67) Lang, M.; Werner, M.; Dockhorn, R.; Kreer, T. Arm Retraction Dynamics in Dense Polymer Brushes. Macromolecules 2016, 49, 5190-5201.

(68) Zhang, W.; Gomez, E. D.; Milner, S. T. Surface-Induced Chain Alignment of Semiflexible Polymers. Macromolecules 2016, 49, 963-971. 


\section{Graphical TOC Entry}

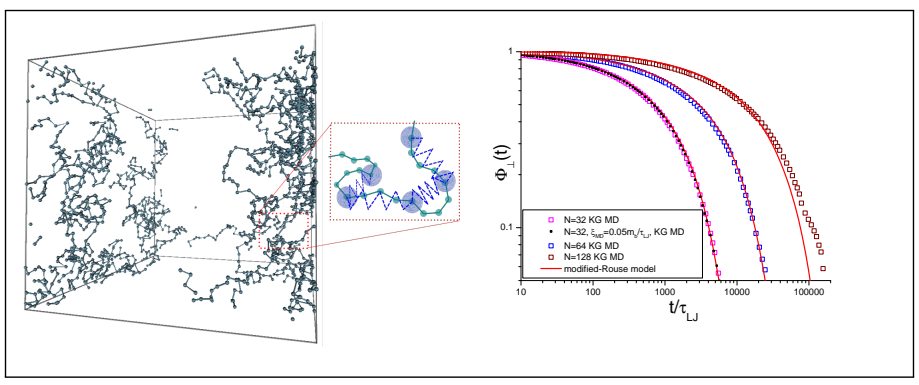

